Annales Geophysicae (2002) 20: 917-935 (c) European Geophysical Society 2002

\title{
Arrival times of Flare/Halo CME associated shocks at the Earth: comparison of the predictions of three numerical models with these observations
}

\author{
S. M. P. McKenna-Lawlor ${ }^{1}$, M. Dryer ${ }^{2,3}$, Z. Smith ${ }^{3}$, K. Kecskemety ${ }^{4}$, C. D. Fry ${ }^{2}$, W. Sun $^{5}$, C. S. Deehr ${ }^{5}$, \\ D. Berdichevsky ${ }^{6,7}$, K. Kudela ${ }^{8}$, and G. Zastenker ${ }^{9}$ \\ ${ }^{1}$ Space Technology Ireland, National University of Ireland, Maynooth, Co. Kildare, Ireland \\ ${ }^{2}$ Exploration Physics International, Inc., Milford, New Hampshire, 03055, USA \\ ${ }^{3}$ NOAA Space Environment Center, Boulder, Colorado, 80305, USA \\ ${ }^{4}$ KFKI Research Institute for Particle and Nuclear Physics, Budapest, Hungary \\ ${ }^{5}$ Geophysical Institute, University of Alaska, Fairbanks, Alaska, 99775, USA \\ ${ }^{6}$ Emergent Information Technologies, Inc., Largo, Maryland, 20774, USA \\ ${ }^{7}$ Laboratory for Extraterrestrial Physics, NASA, GSFC, Code 690, Greenbelt, Maryland, 20771, USA \\ ${ }^{8}$ Institute for Experimental Physics, Kosice, Slovakia \\ ${ }^{9}$ Space Research Institute, Moscow, 117997, Russia
}

Received: 11 September 2001 - Revised: 4 March 2002 - Accepted: 11 March 2002

\begin{abstract}
The arrival times at L1 of eleven travelling shocks associated both with X-ray flaring and with halo CMEs recorded aboard SOHO/LASCO have been considered. Close to the Sun the velocities of these events were estimated using either Type II radio records or CME speeds. Close to the Earth the shocks were detected in the data of various solar wind plasma, interplanetary magnetic field (IMF) and energetic particle experiments aboard SOHO, ACE, WIND, INTERBALL-1 and IMP-8. The real-time shock arrival predictions of three numerical models, namely the Shock Time of Arrival Model (STOA), the Interplanetary Shock Propagation Model (ISPM) and the Hakamada-Akasofu-Fry Solar Wind Model (HAFv.2) were tested against these observations. This is the first time that energetic protons (tens of $\mathrm{keV}$ to a few $\mathrm{MeV}$ ) have been used to complement plasma and IMF data in validating shock propagation models. The models were all generally successful in predicting shock arrivals. STOA provided the smallest values of the "predicted minus measured" arrival times and displayed a typical predictive precision better than about $8 \mathrm{~h}$. The ratio of the calculated standard deviation of the transit times to Earth to the standard deviation of the measurements was estimated for each model (treating interacting events as composite shocks) and these ratios turned out to be $0.60,1.15$ and 1.02 for STOA, ISPM and HAFv.2, respectively. If an event in the sample for which the shock velocity was not well known is omitted from consideration, these ratios become $0.36,0.76$ and 0.81 , respectively. Larger
\end{abstract}

Correspondence to: S. M. P. McKenna-Lawlor (stil@may.ie) statistical samples should now be tested. The ratio of the in situ shock velocity and the "Sun to L1" transit velocity $\left(V_{s h} / V_{t r}\right)$ was in the range of $0.7-0.9$ for individual, noninteracting, shock events. HAFv.2 uniquely provided information on those changes in the COBpoint (the moving Connection point on the shock along the IMF to the OBserver) which directly influenced energetic particle rise times. This model also illustrated the non-uniform upstream conditions through which the various shocks propagated; furthermore it simulated shock deformation on a scale of fractions of an AU. On the spatial scale $\left(300 R_{E}\right)$, where near-Earth spacecraft are located, the passing shocks, in conformity with the models, were found to be locally planar. The shocks also showed tilting relative to the Sun-Earth line, probably reflecting the inherent directionality associated with their solar origin.

Key words. Interplanetary physics (energetic particles; interplanetary shocks; solar wind plasma)

\section{Introduction}

Space weather results from dynamic changes in the solar atmosphere which, by virtue of the associated propagation of shocks and energetic particles through the interplanetary medium, produces consequences in the terrestrial environment that can be inimical to life as well as deleterious to telecommunications. Also, the performance and reliability of a wide variety of space-borne and ground-based technological systems can be adversely effected by fast propagating 
solar related disturbances, the transitory nature of which has led to their being generally referred to as space weather.

The disturbances stimulated when certain coronal mass ejection (CME) related shocks reach the Earth, constitute one of the most important aspects of space weather. These CMEs, together with their preceding interplanetary (I) shock waves, are sometimes referred to as ICMEs, due to their large-scale structures within the heliosphere (Dryer, 1994). The goal of the present paper is to test real-time prediction algorithms of the arrival of CME shocks at the Earth. We have selected as progenitors "halo CMEs" which are seen as expanding bright "rings" around the Sun and are thus likely to impact the Earth. The (mass) ejection itself is confined inside a smaller volume than that encompassed by the associated shock and, if it originates at a solar longitude far from the Central Meridian, it may well miss the Earth.

It has been proposed that neither these CMEs nor their shocks are related in any fundamental way to solar flares (Kahler, 1992; Gosling, 1993; Gosling and Hundhausen, 1995 and references therein). This view, the "solar flare myth", has been challenged by a number of workers (cf. Dryer, 1996; Svestka, 1995, 2001 and others), who argue that CME connected causes are at least bimodal in their solar magnetic complexities. In this latter scenario, small-scale active region flaring, as well as the large-scale helmet-streamer destabilizations exclusively cited in the former view, should be included among possible CME "causes". Recent white light and extreme ultraviolet observations made using the SOHO/LASCO coronagraphs and EIT imaging telescopes during the maximum phase of Solar Cycle 23 have been reported by Andrews and Howard (2001) to provide evidence in support of a "bimodal" picture. The present paper is concerned with a sample of flare-associated halo CMEs, their shocks and the particles that accompanied them.

Geo-effective CMEs are often associated with a large value of $\boldsymbol{B}_{z}$ (the magnetic field component perpendicular to the ecliptic plane) which is in addition southward directed and endures for several hours. In such circumstances, energy from the solar wind can be more easily transferred to the magnetosphere of the Earth than is the case at other times, due to the associated possibility of efficient reconnection between the IMF (Interplanetary Magnetic Field) and the geomagnetic field at the dayside magnetopause. CMEs will usually not produce significant geomagnetic storms when the southward $\boldsymbol{B}_{z}$ is small, if $\boldsymbol{B}_{z}$ is directed northward or if the ICME source on the Sun is unfavorably far from the Central Meridian. This topic, in particular the shock within the ICME context (see above), is examined in detail by Gonzalez et al. (1994, 1999).

At the time of writing, initial CME speeds can be measured only in the plane-of-the-sky (POS). Thus, the Earth directed component of the speeds can only be estimated. The ICME coronal shock speed, however, can be approximated from metric radio Type II frequency drifts. Reliance is primarily made in the present paper on these latter shock speed estimates.
Substantial magnetic energy is released in association with solar flare events accompanied by CMEs. Particles accelerated to high energies by CME shocks display characteristic, gradually rising, profiles (Kahler et al., 1984; Kahler, 1992; Gosling, 1993; Reames, 1995, 1997; Reames and Ng, 1996; Lario et al., 1998) (see, also Sects. 5.5 and 5.6.). Measurements of the ionization rates of $F e$ in such solar ejecta suggest that ambient (unheated) coronal material provides the seed population for the constituent particles, even those that are shock accelerated to energies up to $\sim 600 \mathrm{MeV} / \mathrm{amu}$ (Tylka et al., 1995). If the shocks that generate such "Gradual" Solar Energetic Particle (SEP) events are sufficiently energetic and their propagation direction is appropriate, these particles can reach the Earth. An SSC (Sudden Storm Commencement) provides in more than $90 \%$ of these cases a clear indication of the arrival of such a shock at the Earth (see also above).

It is noted that "Impulsive" SEPs, which in contrast have their origin in compact Impulsive Flares, are ${ }^{3} \mathrm{He}$ rich. Also, it is believed that the one thousand fold enhancement in the ratio ${ }^{3} \mathrm{He} /{ }^{4} \mathrm{He}$ that characterizes these events originates in resonant wave-particle interactions in the flare plasma (e.g. Reames, 1990, 1999; Roth and Temerin, 1997). Further, ${ }^{3} \mathrm{He} /{ }^{4} \mathrm{He}$ enhancements detected during several interplanetary shock events by Popecki et al. (2001) using the Advanced Composition Explorer/Ultra Low Energy Isotope Spectrometer ACE/ULEIS, have been interpreted by Desai et al. (2001) to constitute re-accelerated suprathermal ions originating in flare events near the Sun.

\subsection{The present study}

In the present paper, the arrival times at the Earth of eleven flare associated, halo CME generated, shocks are forecast based on "real-time" data using three models, namely the Shock Time of Arrival Model (STOA), the Interplanetary Shock Propagation Model (ISPM) and the HakamadaAkasofu-Fry Solar Wind Model (HAFv.2). These predictions are thereafter compared with the measured arrival times. The shocks were detected during their passage near L1 in plasma and magnetic field, as well as in energetic particle data recorded aboard SOHO, ACE, WIND, IMP-8 and INTERBALL-1. (L1 is the first Lagrangian Point based on the three body dynamical problem located at $0.99 \mathrm{AU}$ on the Sun-Earth line, just ahead of, and in synchronous orbit with, the Earth as it moves around the Sun.)

This is the first time that energetic proton data have been used to complement solar wind plasma and interplanetary magnetic field (IMF) observations in validating the predictions of numerical modeling. It is noted that the distance between L1 and the Earth is smaller than the resolution of the numerical models used. Also, among the spacecraft available, only SOHO and ACE remained permanently close to L1, while the GSE positions of, for example, WIND were actually far from L1 during six of the events considered. For practical purposes, the arrival of a shock at L1 may be taken as equivalent to its arrival at the Earth. The comparisons 
Table 1. Characteristics of eleven solar flares with accompanying Metric Type II and Halo CMEs

\begin{tabular}{|c|c|c|c|c|c|c|c|c|c|c|}
\hline \multirow{3}{*}{$\begin{array}{c}1 \\
\text { Event } \\
\text { No. }\end{array}$} & \multirow{3}{*}{$\begin{array}{c}2 \\
\text { Date } \\
\text { DDMMYY }\end{array}$} & 3 & 4 & \multirow{3}{*}{$\begin{array}{c}5 \\
\text { Max.(UT) } \\
\text { X-ray }\end{array}$} & \multirow{3}{*}{$\begin{array}{c}6 \\
\text { Flare } \\
\text { class }\end{array}$} & \multirow{3}{*}{$\begin{array}{c}7 \\
\text { Flare } \\
\text { Location }\end{array}$} & \multirow{3}{*}{$\begin{array}{c}8 \\
\tau \\
(\mathrm{h})\end{array}$} & \multirow{3}{*}{$\begin{array}{c}9 \\
\mathbf{V}_{\mathbf{s}}(\mathbf{I I}) \\
(\mathrm{km} / \mathrm{s})\end{array}$} & \multirow{3}{*}{$\begin{array}{c}10 \\
\mathbf{V}_{\mathbf{c m e}} \\
(\mathrm{km} / \mathrm{s})\end{array}$} & \multirow{3}{*}{$\begin{array}{c}11 \\
\mathbf{T}_{\text {cme }}-\mathbf{T}_{\mathbf{I I}} \\
(\mathrm{h}: \mathrm{m})\end{array}$} \\
\hline & & \multicolumn{2}{|c|}{ Start (UT) } & & & & & & & \\
\hline & & II & CME & & & & & & & \\
\hline 1 & $12 / 05 / 97$ & $05: 16$ & $06: 30$ & $05: 16$ & $\mathrm{C} 1 / 1 \mathrm{~F}$ & N21 W08 & 2.50 & 1400 & 306 & $1: 14$ \\
\hline 2 & $04 / 11 / 97$ & 06:08 & $06: 10$ & $05: 54$ & $\mathrm{X} 2 / 2 \mathrm{~B}$ & S14 W33 & 1.25 & 1400 & 830 & $0: 02$ \\
\hline 3 & $02 / 05 / 98$ & $13: 42$ & $14: 56$ & $13: 42$ & X1/3B & S15 W15 & 1.00 & $2000^{*}$ & 1044 & $1: 14$ \\
\hline 4 & $17 / 02 / 00$ & $18: 52$ & $20: 06$ & $18: 52$ & M2/1B & S25 W16 & 0.66 & 700 & 550 & $1: 14$ \\
\hline 5 & $17 / 02 / 00$ & $20: 25$ & $21: 30$ & $20: 35$ & $\mathrm{M} 1 / 2 \mathrm{~N}$ & S29 E07 & 1.17 & 550 & $\sim$ & 1:06 \\
\hline 6 & $04 / 04 / 00$ & $15: 25$ & $16: 32$ & $15: 41$ & $\mathrm{M} 1 / 2 \mathrm{~F}$ & N16 W66 & 1.00 & 2000 & 984 & 1:07 \\
\hline 7 & 06/06/00 & $15: 23$ & $15: 54$ & $15: 23$ & $\mathrm{X} 2 / 3 \mathrm{~B}$ & N20 E13 & 1.50 & 1189 & 908 & $0: 31$ \\
\hline 8 & $14 / 07 / 00$ & $10: 20$ & $10: 54$ & $10: 24$ & X6/3B & N22 W07 & 1.50 & $1800 *$ & 1775 & $0: 34$ \\
\hline 9 & $20 / 01 / 01$ & $18: 42$ & $19: 31$ & $18: 47$ & $\mathrm{M} 1 / \mathrm{SF}$ & S07 E40 & 0.67 & 700 & 673 & $0: 50$ \\
\hline 10 & 20/01/01 & $21: 14$ & $21: 54$ & $21: 20$ & M8/2B & S07 E46 & 1.00 & 1300 & 1576 & $0: 40$ \\
\hline 11 & $28 / 01 / 01$ & $16: 00$ & $15: 54$ & $16: 00$ & M1.5/? & S04 W59 & 1.00 & $1000 * *$ & 795 & $-0: 06$ \\
\hline
\end{tabular}

$\begin{array}{ll}\mathrm{V}_{\mathrm{cme}} & \text { velocity of the CME (private communication, from http://lasco-www.nrl.navy.mil/cmelist.html); } \\ \text { start time of the CME (Column 4) is from real-time reports. } \\ \text { velocity } V_{S} \text { is heuristically based on } V_{\mathrm{cme}} \text {, not derived from metric Type II. } \\ \sim \\ \text { CME merged with the preceding CME as implied by “...part of preceeding CME was visible above } \\ \text { S-pole from as early as 20:06 UT" (Simon Plunkett, private communication, 2000). } \\ V_{S} \text { estimated from WIND/WAVES (kilometric Type II measurements). }\end{array}$

Column 1: Sequential event numbers.

Column 2: Day, month and year.

Cols. 3-4: Observed starting times (UT) of each related metric Type II and halo CME.

Column 5: Corresponding times of peak soft X-ray flux.

Column 6: X-ray and optical flare classifications.

Column 7: $\quad$ Solar disk locations.

Column 8: The proxy piston-driving time (tau) of each shock as it moved at the Type II speed.

Column 9: $\quad$ Shock velocities based on Type II radio bursts.

Column 10: Real-time velocities of their shock-related CMEs.

Column 11: CME start above LASCO/C2 (2 Rs) minus metric Type II start time.

made provide insights into the strengths and weaknesses of the individual models in predicting, for space weather applications, shock arrivals at the Earth and allow us to suggest how the forecasting procedure might be improved.

In Sect. 2, the eleven solar events producing the traveling shocks studied herein are described. In Sect. 3, details of the three individual models used in providing the predictive data are presented. In Sect. 4, the measured and predicted shock arrival times of each of the events in our sample are intercompared and several representative cases are analyzed in detail. In Sect. 5, the results of the study are discussed within the context of other relevant shock investigations in the literature. General conclusions are presented in Sect. 6.

\section{Observations at the Sun as an input to real-time fore- casting}

Table 1 provides a list of eleven halo CMEs recorded by the Large Angle Spectroscope Coronagraph (LASCO) aboard
SOHO. This instrument is described in detail in Brueckner et al. (1995). The table also displays data concerning their related metric Type II and soft X-ray events (the table footnotes give a detailed list of the entries column by column).

Type II burst data (Column 3) are available in real time at metric wavelengths from the United States Air Force Radio Solar Telescope Network (RSTN) which, at the time of the first two events chosen for analysis, used the bandwidth 25$85 \mathrm{MHz}$ at Palehua, Hawaii; San Vito, Italy; Sagamore Hill, Massachusetts and Learmonth, Australia. Upgraded digital Solar Radio Spectrographs (SRS) have extended more recently the bandwidth to $25-180 \mathrm{MHz}$ at Palehua, San Vito and Learmonth. The RSTN and SRS data are complemented by metric radio measurements made at the Ionospheric Prediction Service in Culgoora, Australia.

The starting time of a halo CME (Column 4) is taken as the time when the event considered was first detected by the LASCO experimenters (at two solar radii) above the LASCO/C2 occulting disk. Optical flare identification 
(Column 7) is used in determining the location of the source.

Column 8 provides the so-called (proxy) piston-driving time tau $(\tau)$ of the shock as it moves at the Type II speed. This latter temporal duration is estimated using GOES 1-8 Angstrom soft X-ray temporal profiles (the full width at half maximum measured linearly on the log plot from just above the pre-event background flux level).

The shock velocities $V_{s}$ (II), Column 9, are derived from drifting (metric) Type II bursts reported in real time to NOAA by various radio observatories. Several coronal density models are available for use when calculating the velocities concerned. The density model used by the US Air Force RSTN sites is an empirical factor of $1 \times$ the Quiet Sun Newkirk model (Newkirk, 1961). The Australian IPS site at Culgoora also uses $1 \times$ Newkirk.

Column 11 shows that the "CME start" (as defined above) is almost always later than the metric Type II start time. This latter time is usually close to the time of maximum X-ray flux and, therefore, closer to the start time of the physical process initiating the flare.

Those cases where the metric Type II shock speeds $\left(V_{s}\right)$ were estimated are marked with an asterisk. An estimate was required when the reported Type II speed was either in question or not reported. In both of these circumstances, the shock speed was estimated on the basis of the velocity of the CME in the Plane-of-Sky (POS), as measured by the LASCO experimenters in real time (provided by S. Plunkett and K. Schenk, private communications between 1997 and 2001). These speeds were determined within the LASCO team by applying a height vs. time technique to the CME images recorded using two coordinated onboard coronagraphs that image the solar atmosphere between 2.0-30 $R_{s}$ (where $R_{S}$ is one solar radius, $\left.6.95 \times 10^{5} \mathrm{~km}\right)$. Decametric shock speeds from WIND/WAVES data (M. Kaiser and M. Reiner, private communication 2000) are complementary to the metric measurements, but were not generally available for realtime forecasting.

There were three other circumstances in which estimated rather than measured values were potentially used. These three situations and the procedures for handling them are as follows:

(i) If the flare site was near Central Meridian and a Type II burst with speed $V_{s}$ was reported, but $V_{s}$ was less than the reported fast $V_{\text {cme }}(\sim 1500 \mathrm{~km} / \mathrm{s})$ in the POS, then the shock was inferred to be quasi-spherical and to display above the flare site the velocity of the CME.

(ii) If the flare site was near the limb and again $V_{s}$ was $<V_{\text {cme }}$, then, as in the previous case, the reported $V_{\text {cme }}$ was taken to represent the shock speed (i.e. it was assumed that the leading edge of the white light CME was coincident with the shock surface). In this case, the lower speed $V_{S}$ which was not used was assumed to represent the lower, slower, coronal speed of the selfsimilarly expanding quasi-hemispherical shock (Dryer, 1974). (iii) If the flare was near the limb but $V_{s}$ was $>V_{\text {cme }}$, then the Type II value was used. In this case the velocity of the CME was usually lower than $1500 \mathrm{~km} / \mathrm{s}$.

\section{The numerical models}

Three widely used models, namely the Shock Time of Arrival Model (STOA), the Interplanetary Shock Propagation (ISPM) Solar Wind Model and the Hakadada-Akasofu-Fry (HAFv.2) Model were employed in real time to predict the arrival time at L1 of each of the eleven measured travelling shocks associated with the solar events listed in Table 1. An overview of the predictive models is provided below.

\subsection{The Shock Time of Arrival Model (STOA)}

The STOA Model is based on the similarity theory of blast waves (modified by the piston-driving concept) that emanate from point explosions. The initial explosion (flare) drives a shock at what is assumed to be a constant speed $\left(V_{S}\right)$ for a specified length of time $\tau$ estimated using X-ray duration measurements. Details are given by Dryer (1974); Dryer and Smart (1984); Smart et al. (1984, 1986); Smart and Shea (1985); Lewis and Dryer (1987) and Smith, Dryer and Armstrong (1993).

The shock, thereafter, decelerates to a blast wave as it expands outwards (with $V_{s} \sim R^{-1 / 2}$, where $R$ is the heliocentric radius). The magnitude of the total energy conversion process determines the solid angle of quasi-spherical shock propagation as well as how far a particular shock will propagate as it "rides over" a uniform background solar wind. The fastest part of the shock is assumed to be nearly coincident with the heliocentric radius vector aligned from the center of the Sun through the flare site. Viscous and ohmic dissipation is inferred to cause the flanks of the shock to decay first to a magnetohydrodynamic wave. The shock speed directly above the flare is calculated (see above) from the Type II radio frequency drift rate (which is proportional to the radial gradient of the square root of the assumed local coronal electron density).

The driver, therefore, is initiated when the kinetic energy pulse begins, and it ends after $\tau$ expires. Other drivers considered in the literature include pulses of density, temperature, momentum and magnetic energy (cf. reviews by Dryer, 1974, 1994). Some workers (cf. Odstrcil et al., 1996; Odstrcil and Pizzo, 1999) have referred to the subsequent history of the affected plasma (during the input duration $\tau$ ) in its heliospheric journey as the "CME", the boundaries of which are the contact surfaces (or piston) at the start and end of $\tau$. The relationship, however, between near-Sun white light CMEs and these ICMEs (including their shocks) has yet to be satisfactorily elucidated.

STOA uses a cosine function to account for the longitudinal dependence of the shock geometry in the ecliptic plane (following empirical studies by Lepping and Chao, 1976). The shock speed is assumed to decrease from maximum in 
the direction of the flare via this cosine function to produce a non-spherical shape in longitude. This spatially dependent shock speed is taken to be constant during the piston-driving phase. The longitudinal cosine function is maintained during the decelerating blast wave phase. When the modeled shock reaches the observer's position (anywhere in the ecliptic plane), its speed relative to a representative, uniform background, solar wind is used to compute the local magnetoacoustic Mach number Ma. The resulting number provides an indication of the expected shock strength.

\subsection{The Interplanetary Shock Propagation Model (ISPM)}

The Interplanetary Shock Propagation Model (ISPM) is based on a $2.5 \mathrm{D}$ magnetohydrodynamic parametric study of numerically simulated shocks that shows the organizing parameter to be the net energy input to the solar wind (Smith and Dryer, 1990). If the net energy injected into the solar wind by a source on the Sun is either known or estimated, then the transit time and strength of the shock at $1 \mathrm{AU}$ can be computed using algebraic equations developed during the original parameterization study. It is noted that, in the case of drivers of duration greater than two hours, the properties of the leading shock remain unchanged. Therefore, drivers longer than two hours are, in practice, automatically truncated to two. When the modeled shock reaches the Earth, the logarithm (base 10) of the normalized dynamic pressure jump in a uniform, representative background, solar wind provides the Shock Strength Index (SSI). This index is used to determine whether a shock is expected at the Earth or if it will have already decayed to an MHD wave. The aim, as for STOA, is to provide an indication of the expected shock strength. For further details, see Smith and Dryer (1995).

\subsubsection{Observational data inputs to the STOA and ISPM models}

Both the STOA and ISPM models require the initial coronal shock velocity, the input energy duration and the location of the source on the Sun as input observational data. The shock velocities are generally derived at several reporting sites from drifting (metric) Type II bursts. In some cases, plane of sky (POS) speeds measured by the SOHO/LASCO experimenters, which are forwarded in real time to NOAA, are used (see Sect. 2). The shock velocities derived from radio burst measurements are reported to NOAA and issued as alerts in real time. Velocities calculated by the radio observers using $1 \times$ the Quiet Sun Newkirk model (Newkirk, 1961) were used for each of the events considered in the present study. It is noted that these "real-time" values can be changed when the measurements are finally listed in The National Oceanographic and Atmospheric Administration / National Geophysical Data Center (NOAA/NGDC) publication Solar Geophysical Data (Comprehensive Reports).

The STOA model requires. in addition. the "Parkertype" spherically symmetric and polytropic solar wind velocity profile up to $1 \mathrm{AU}$. This value is obtained from real- time (L1) satellite data, and it is assumed that a uniform solar wind (in heliolongitude) is present upstream of the shock. A default value of $400 \mathrm{~km} / \mathrm{s}$ is taken if real-time data are unavailable. When a shock arrives at the Earth, however, it has already passed through plasma that will not itself reach the Earth (on average) for several days. Therefore, solar wind measurements do not significantly help the predictions and their use in forecasting is less of an advantage than might at first appear.

ISPM is based on a single background solar wind model that asks the user whether there has been a prior event within the previous $24 \mathrm{~h}$. If the answer is affirmative, a cautionary note is sounded concerning the prediction, due to the possibility that temporally and/or spatially close solar wind events will interact. Both STOA and ISPM are based on the assumption that solar initiated shocks do not interact en route to L1. Furthermore, both of these models do not (as just indicated above) consider upstream stream-stream interactions.

\subsubsection{STOA and ISPM outputs}

Both models predict whether a shock will arrive at the Earth and, if so, when. They also provide a measure of the shock strength. STOA gives the shock magneto-acoustic Mach number, Ma, while ISPM yields a Shock Strength Index, SSI, which identifies shocks that are too weak to be significant when they reach L1. Thus, the value SSI $=0$ is taken to provide a threshold value equivalent to the limit $\mathrm{Ma}=1.0$, below which shocks decay to MHD waves.

\subsection{The Hakamada-Akasofu-Fry (HAFv.2) solar wind model}

HAFv.2 (HAF version 2) is an update of an earlier model HAFv.1 (Akasofu and Fry, 1986), the history of which is described by Fry et al. $(2001 \mathrm{a}, \mathrm{b})$. It is kinematic in that it kinetically projects the flow of the solar wind from inhomogeneous sources near the Sun out into interplanetary space. It is modified in that the model adjusts the flow for streamstream interactions as faster streams overtake slower ones. More importantly, solar surface magnetograms are projected via the assumptions of Potential Theory onto the source surface at $R=2.5 R_{s}$, to provide both radial magnetic field and solar wind speed as an input to HAFv.2 (Arge and Pizzo, 2000).

This modified kinematic model constitutes a compromise between realistic modeling of solar wind conditions in interplanetary space and the need for "real-time" predictions. HAFv. 2 models successfully those gross features of the solar wind that are the most important for geo-effectiveness.

Whereas MHD solutions integrate the equations of motion to obtain velocity, the kinematic model begins with these equations integrated twice to yield the fluid parcel positions. Velocity then comes from $d x / d t$. HAFv. 2 is thus configured both for the ambient and for the event driven solar wind (see below). 


\subsubsection{The ambient solar wind}

The ambient background solar wind is established by the initial conditions of the model at its inner computational boundary $\left(2.5 R_{S}\right)$. In this scenario, the outflow of plasma from a rotating inhomogeneous source surface at 2.5 solar radii from the center of the Sun is assumed to be radial. The IMF lines are deemed, meanwhile, to have their foot-points anchored to the source surface and to be dragged along with the plasma flow. The rotation of the Sun and the frozen-field condition results in a garden-hose pattern (the "Parker Spiral") when the IMF lines are displayed in the ecliptic plane. Alternating slower and faster streams are emitted from the Sun along fixed radials as the rotating source region sweeps past. If the speed differential is great enough, Co-rotating Interaction Regions (CIRs) and even shock interfaces may form.

\subsubsection{The event driven solar wind}

Energy is deemed to be input at the inner computational boundary during solar flares. Electromagnetic measurements (see above) provide information on the start time and later evolution of this energy source, as well as on its disk location and estimated size. Disturbance energy is made manifest by enhanced solar wind speed at the source surface. The resulting compression (Event Driven Component) is represented by time-dependent stream boundaries. The development of a stream-stream interaction may lead to the production of shocks.

\subsubsection{Observational inputs to the HAFv.2 model}

HAFv. 2 generally uses the same observational inputs as STOA and ISPM, but differs from them in the way the background solar wind is treated. STOA utilizes the observed solar wind speed at L1, while ISPM employs a fixed internal model with a representative speed of $360 \mathrm{~km} / \mathrm{s}$ at $1 \mathrm{AU}$. HAFv.2, on the other hand, models the inhomogeneous ambient solar wind that affects the propagation of disturbances en route from the Sun to the Earth. Realistic inner boundary conditions determine the modeled background solar wind flow and the IMF topology. These data are derived from synoptic solar source surface maps of the radial magnetic field and from calculations of the magnetic flux divergence and solar wind velocity close to the Sun (Arge and Pizzo, 2000). The latter information is updated and made available daily on the NOAA Space Operations website: http: //www.sec.noaa.gov.

\subsubsection{HAFv.2 Outputs}

HAFv. 2 predicts the solar wind speed, density, dynamic pressure and the IMF vector as functions of time at any point in the heliosphere. The present application is relevant to L1/Earth. The temporal profile of the predicted dynamic pressure at L1 is used to compute a Shock Searching Index (SSI), in a similar fashion to the computation of the ISPM Shock Strength Index (Sect. 3.2.). A predicted shock ar- rival time is generated when this index exceeds an empirical threshold (set at -0.5 ) and the shock is considered to be significant when the predicted post-shock dynamic pressure is greater than its pre-shock value, thereby requiring the establishment of a second empirical threshold (set at $8 \mathrm{nPa}$ ).

\section{Comparison between the predicted times of arrival at L1 of solar flare initiated shocks based on three dif- ferent models and their measured arrival times}

In Table 2, the predicted times of arrival (Columns 9, 11 and 13) of 11 shocks associated with the halo CME flares listed in Table 1, are compared with their measured arrival times (see Columns 3 and 8).

In the case of STOA and ISPM, the predicted arrival times comprise estimates made in real time by the forecasters (MD and ZS) at NOAA, based on quick-look X-ray, dynamic radio and CME data. These predictions, when they were originally made (1997-2001) were circulated immediately to a wide range of space weather users. In the case of several of the events in the table, real-time predictions were also simultaneously circulated internationally, based on the HAFv.1 model (Akasofu and Fry, 1986). In order to provide state-of-the-art computations for the present study, these latter events were rerun using the (upgraded) HAFv.2 model (Sect. 3.3) and the predictions thus made are listed in Table 2, Column 13, in lieu of the original forecasts based on HAFv.1 The shock arrival predictions concerned are treated here (as in the case of the STOA and ISPM predictions) as "real-time" results, and no attempt was made by the authors to "fit" the predictions to the retrospectively known arrival times.

We consider next whether a shock was predicted within a period of $100 \mathrm{~h}$, starting with the commencement of each of the flare associated metric Type II bursts at the Sun. Using the jargon of predictive modeling procedures routinely adopted at the National Oceanographic and Atmospheric Administration/Space Environment Center (NOAA/SEC), the following terms are defined, following Schaefer (1990):

HIT: Shock predicted within $\pm 24 \mathrm{~h}$ of its detection time;

MISS: Shock detected, but predicted at a time more than $24 \mathrm{~h}$ before or after this detection, or not predicted at all;

FALSE ALARM: Shock not detected but predicted (this case did not occur in our sample);

CORRECT NULL: Shock not detected and not predicted.

The threshold values for predicting an event in the present study were chosen as follows:

- The Mach Number for STOA must be >1.0;

- The Shock Strength Index for ISPM must be $>0.0$; 
Table 2. Observed interplanetary shocks and the corresponding arrival predictions of three numerical models

\begin{tabular}{|c|c|c|c|c|c|c|c|c|c|c|c|c|c|c|c|c|c|c|c|}
\hline \multirow{4}{*}{$\begin{array}{c}1 \\
\text { Event } \\
\text { No. }\end{array}$} & 2 & 3 & 4 & 5 & 6 & 7 & 8 & 9 & 10 & 11 & 12 & 13 & 14 & 15 & 16 & 17 & 18 & 19 & 20 \\
\hline & \multicolumn{6}{|c|}{ IP Shock } & & \multicolumn{2}{|c|}{ STOA } & \multicolumn{2}{|c|}{ ISPM } & \multicolumn{2}{|c|}{ HAFv.2 } & $\Delta \mathrm{Ts}$ & $\Delta \mathrm{Ti}$ & $\Delta \mathrm{Th}$ & \multirow{2}{*}{\multicolumn{3}{|c|}{$\begin{array}{c}\text { Contingency } \\
\text { Table-info }\end{array}$}} \\
\hline & Date & & $\mathrm{S} / \mathrm{C}$ & $V_{t r}$ & $V_{s h}$ & $V_{s h} / V_{t r}$ & TTd & TTs & Ma & TTi & SSI & TTh & SSI & \multicolumn{3}{|c|}{ pred.-obs. } & & & \\
\hline & DDMMYY & UT & & $(\mathrm{km} / \mathrm{s})$ & $(\mathrm{km} / \mathrm{s})$ & & (h) & (h) & & (h) & & (h) & & (h) & (h) & (h) & $\mathbf{S}$ & I & H-v.2 \\
\hline 1 & $15 / 05 / 97$ & $01: 15$ & W & 613 & 467 & 0.76 & 68 & 46 & 7 & 30 & 1.3 & 37 & 0.13 & -22 & -39 & -31 & $\mathrm{~h}$ & $\mathrm{~m}$ & $\mathrm{~m}$ \\
\hline 2 & 06/11/97 & $22: 18$ & W & 651 & 495 & 0.76 & 64 & 52 & 4.8 & 40 & 0.9 & 41 & 0.01 & -12 & -24 & -23 & $\mathrm{~h}$ & $\mathrm{~h}$ & $\mathrm{~h}$ \\
\hline 4 & $20 / 02 / 00$ & $20: 47$ & $\mathrm{~A}$ & 563 & 487 & 0.86 & 74 & 75 & 1.5 & 81 & 0.5 & 63 & 0.30 & 1 & 7 & -11 & $\mathrm{~h}$ & $\mathrm{~h}$ & $\mathrm{~h}$ \\
\hline 5 & $"$ & $"$ & $"$ & 579 & $"$ & 0.84 & 72 & 77 & 1.3 & 86 & 0.6 & 62 & 0.30 & in & in & in & $\mathrm{cn}$ & $\mathrm{cn}$ & $\mathrm{cn}$ \\
\hline 6 & $06 / 04 / 00$ & $16: 03$ & A & 850 & 756 & 0.89 & 49 & 46 & 5.6 & 45 & 0.6 & 42 & -0.13 & -3 & -4 & -6.6 & $\mathrm{~h}$ & $\mathrm{~h}$ & $\mathrm{~h}$ \\
\hline 7 & 08/06/00 & $08: 41$ & A & 1016 & 864 & 0.85 & 41 & 49 & 4.3 & 38 & 1 & 33 & 0.20 & 8 & -4 & -8.3 & $\mathrm{~h}$ & $\mathrm{~h}$ & $\mathrm{~h}$ \\
\hline 11 & $31 / 01 / 01$ & $07: 22$ & $\mathrm{~A}$ & 661 & 470 & 0.71 & 64 & 66 & 2.3 & mhd & -0.3 & 71 & -0.04 & 3 & $\mathrm{~m}$ & 8 & $\mathrm{~h}$ & $\mathrm{~m}$ & $\mathrm{~h}$ \\
\hline
\end{tabular}

\footnotetext{
$\mathrm{V}_{\mathrm{tr}} \quad$ Transit velocity $=1 \mathrm{AU} / \mathrm{TTd}$.

$\mathrm{V}_{\mathrm{sh}} \quad$ Local shock velocity at $1 \mathrm{AU}$ (values for Events 1-3, 7, 8 from D. B.: the rest were estimated by Z. S. assuming

$\begin{array}{ll} & \text { radial shocks). } \\ \text { TTd } & \text { Transit time from Sun to Earth. }\end{array}$

The horizontal are used to group the events closely spaced in time (\#4, 5 and \#9, 10) whose interplanetary shocks are expected

lines to interact; "in" denotes interaction; "mhd" decay to an mhd wave. See discussion in text.

" Standard symbol for "same as above".
}

Column 1: $\quad$ Event number as per Table 1.

Cols. 2-4: $\quad$ Date and time of arrival of interplanetary shocks at WIND (W) and at ACE (A).

Cols. 5-7: "Sun to L1" transit velocity $\left(V_{t r}\right)$; estimate of the in situ shock speed $\left(V_{s h}\right)$ and the ratio of these values.

Column 8: The actual shock transit time data (TTd).

Cols. 9-10: The predicted transit times for STOA (TTs) and the modeled magneto-acoustic Mach number (Ma).

Cols. 11-12: $\quad$ The predicted transit times for ISPM (TTi) and the modeled Shock Strength Index (SSI).

Cols. 13-14: The predicted HAFv.2 transit times (TTh) and their corresponding Shock Searching Indices (SSI).

Cols. 15-17: The arrival time errors (predicted minus the observed values) for each of the three models.

Cols. 18-20: Elements of a statistical contingency table for the data set represented by hits (h); misses (m) and correct nulls (cn).

- The Shock Searching Index for HAFv.2 must be > -0.5 and the dynamic pressure jump across the simulated shock must be $>8 \mathrm{nPa}$.

Column 6 of Table 2 provides for each event an estimate of the in situ shock speed $V_{\mathrm{sh}}$. This was determined by measuring the solar wind speed and density (before) and (after) the shock's passage. Conservation of the mass flow across the shock then gives the shock speed $V_{s h}=\left(V_{2} n_{2}-V_{1} n_{1}\right) /\left(n_{2}-\right.$ $\left.n_{1}\right)$.

Columns $15-17$ of Table 2 list the delta values (predicted minus observed arrival time) for the individual models (STOA, ISPM, HAFv.2). Columns 18-20 of the table indicate events that can resultingly be categorized as a HIT (h), a MISS (m) and a Correct Null (cn), according to the above definitions.

It is noted that, when two or more solar events are spatially and temporally close to each other, their associated shocks can potentially interact (Smith et al., 1986). There are two such closely spaced pairs (Events 4 and 5 and Events 9 and 10) in the present sample. Data concerning these pairs are contained between horizontal lines in Table 2.

In seeking to interpret the information contained in Table 2, we will now discuss in detail a representative HIT, a MISS/near MISS and the two paired cases of closely spaced events mentioned above.

\subsection{HIT: Shock Event 7 (8 June 2000)}

On 6 June 2000, a flare located at N20, E13 with its X-ray maximum at 15:23 UT was associated at this same time with a Type II burst. The coronal velocity of the traveling shock was estimated (from metric Type II radio burst drift data) to be $1189 \mathrm{~km} / \mathrm{s}$. The CME speed was $908 \mathrm{~km} / \mathrm{s}$. The shock 


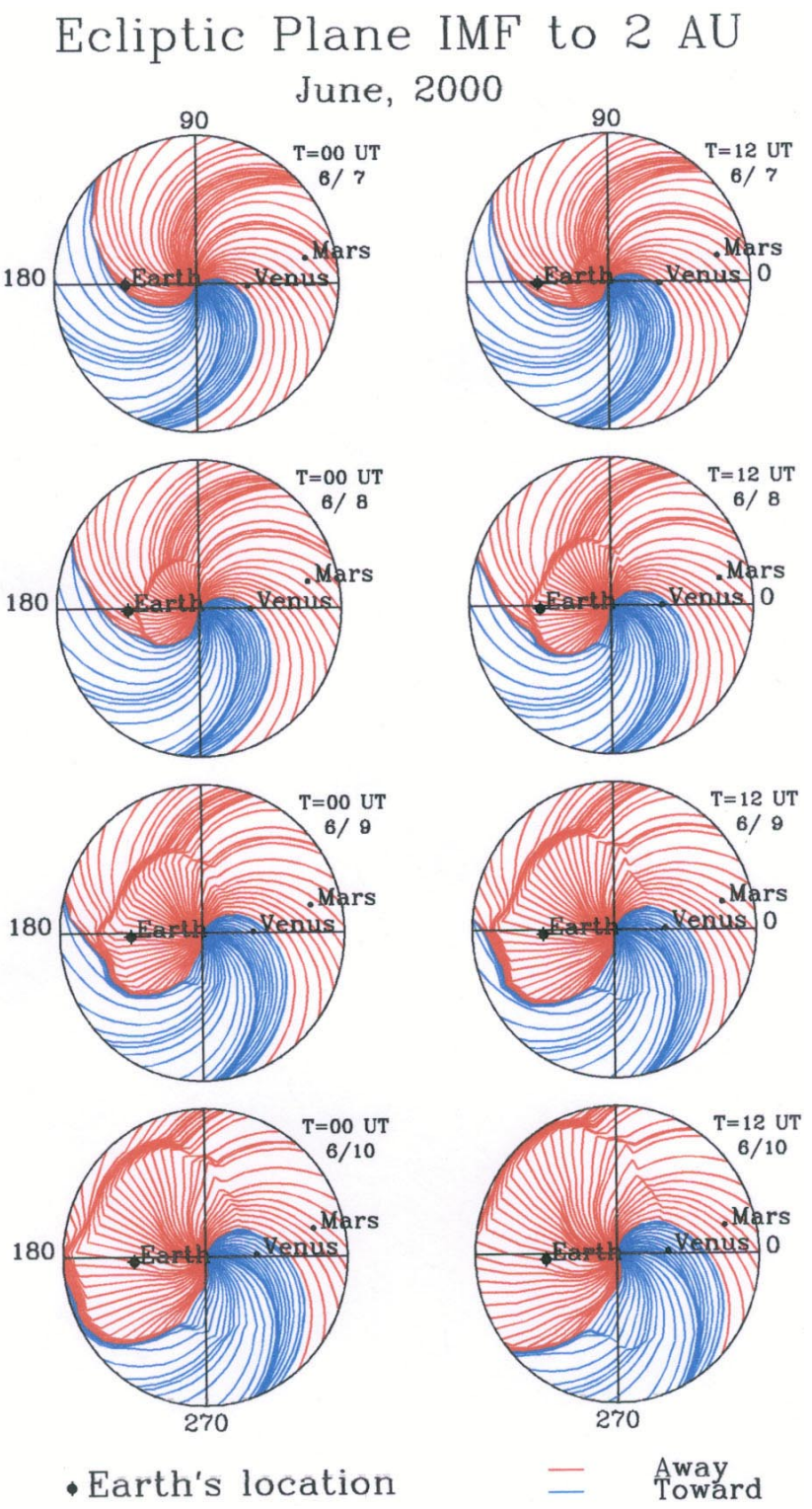

Fig. 1. Ecliptic plane simulation by the HAFv. 2 model of the temporal IMF disturbance caused by the shock from Event 7 (initiated on 6 June 2000). Magnetic field polarity is indicated by blue (toward the Sun) and by red (away). The location of the Earth is indicated by a large black dot; of Venus and Mars, by smaller dots.

was reported at SOHO and at ACE at 08:41 UT on 8 June. The average speed of the shock during its transit was about $1000 \mathrm{~km} / \mathrm{s}$ and the travel time from the Sun to the L1 point was $\sim 41 \mathrm{~h}$.

Figure 1 presents a sequence of HAFv. 2 simulations made in the ecliptic plane from 6 June 2000 at 00:00 UT (i.e. from about $15 \mathrm{~h}$ before the onset of the solar event). Each frame is separated in time by $12 \mathrm{~h}$. The IMF lines are shown in red (away sectors) and blue (towards sectors), representing solar wind streams emanating from open coronal magnetic field regions. The location of the Earth is represented by a large black dot. Venus and Mars are indicated by smaller black dots.

The simulations reveal (top left-hand frame) that the Earth, at heliolongitude $180^{\circ}$, was initially in a "toward" IMF sector (as indicated by the color blue). In Frame 1, it is seen that a sector boundary crossing (heliospheric current sheet passage) occurred on 7 June in association with a well developed CIR. In Frame 2, a shock structure is discernible near the Sun. This can be followed outwards in subsequent frames. The background IMF pattern displays, meanwhile, counter-clockwise rotation in the ecliptic plane.

The center of the leading edge of the modeled shock can be seen to have reached the Earth (Frame 4) several hours prior to 12:00 UT on 8 June. The model shock envelope appears, at that time, to be distorted from its initial, longitudinally symmetric, shape due to its interaction en route with the previously established, non-uniform, interplanetary plasma. In the remaining frames, the shape of the interplanetary disturbance pattern is seen to continue to distort as it propagates beyond $1 \mathrm{AU}$. Note that the eastern flank of the shock has, meanwhile, moved further from the Sun than the western flank, due to its passage through a faster, less dense, solar wind.

The close temporal correspondence between the HAFv.2 predicted and measured shock arrival times $(\Delta \mathrm{Th}=-8.3 \mathrm{~h}$, Table 2, Column 17), together with the graphic representation of solar related interplanetary circumstances contained in Fig. 1, provide confidence that HAFv.2 satisfactorily simulated what transpired. STOA and ISPM also did well with $\Delta \mathrm{Ts}=+8 \mathrm{~h}$ and $\Delta \mathrm{Ti}=-4 \mathrm{~h}$, respectively (Columns 15-16) but without providing the global insight made available in the case of the HAFv. 2 modeling.

\subsection{MISS: Shock Event 1 (15 May 1997)}

On 12 May 1997, an X-ray flare at N21,W08 with maximum at $05: 16 \mathrm{UT}$ had the longest duration $(2 \mathrm{~h} 50 \mathrm{mim})$ of all the events in the present data set. It was associated with a Type II radio burst to which one of the present authors/forecasters (MD) assigned a coronal shock velocity of $1400 \mathrm{~km} / \mathrm{s}$. The SOHO/EIT observers reported an "EIT wave" at 04:50 UT (Thompson et al., 1998). The SOHO/LASCO observers reported the occurrence of a halo $\mathrm{CME}$ at 06:30 UT with a velocity estimated to be $306 \mathrm{~km} / \mathrm{s}$ directly over the solar North Pole. The solar wind velocity at L1 measured at ACE was $300 \mathrm{~km} / \mathrm{s}$. On 15 May at 01:15 UT, an interplanetary shock was recorded in WIND data. Figure 2 presents a composite record made by the LION instrument, described by McKenna-Lawlor et al. (1997) and by the EPHIN (Electron, Proton, Helium INstrument) described by Muller-Mellin et al. (1995), on SOHO in the overall range $\sim 0.3-8 \mathrm{MeV}$ for protons. These data show the passage of the shock at L1 at $\sim 00: 56$ UT. This result is supported by the plasma and magnetic field data shown in the lower three panels of this figure, which were recorded aboard the near-Earth WIND spacecraft. The latter measurements were made by the Solar Wind Experiment (SWE) and by the Magnetic Field Instru- 


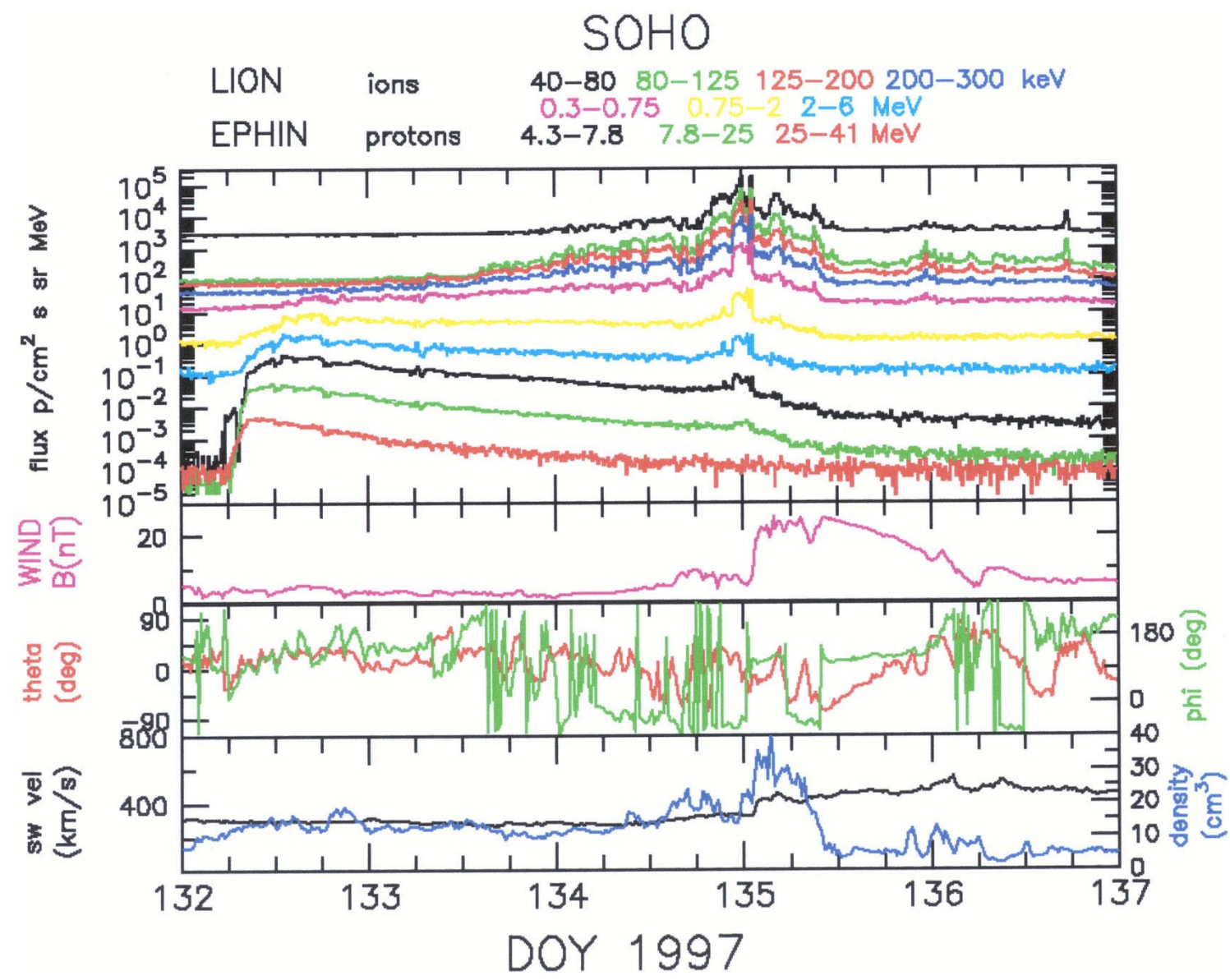

Fig. 2. Top Panel: Energetic particle data recorded by the LION and EPHIN instruments on SOHO during Event 1 (initiated on 12 May 1997). Various energy ranges are indicated by individual colors. The lower three panels present contemporaneous values of the total IMF; polar angles of the IMF and solar wind speed and density measured aboard WIND.

ment (MFI), individually described by Ogilvie et al. (1995) and Lepping et al. (1995).

Predictions generated using the ISPM and HAFv.2 models fell outside the threshold limit of $\pm 24 \mathrm{~h}$ required to score a HIT (see Columns 16 and 17 of Table 2). STOA barely scored a HIT (Column 15). To elucidate this result, it should be considered if the shock speed used in making the prediction was representative. In this regard, it is noted that, from among several reported velocities of the Type II burst $(3500 \pm 500 \mathrm{~km} / \mathrm{s}$ from San Vito, $2500 \pm 500 \mathrm{~km} / \mathrm{s}$ from Learmonth and $1400 \pm 200 \mathrm{~km} / \mathrm{s}$ from Culgoora), the forecaster (noted above) selected the most conservative value of $1400 \mathrm{~km} / \mathrm{s}$. The report from LASCO of an associated Halo CME with $V_{\text {cme }}=306 \mathrm{~km} / \mathrm{s}$ in the POS, supported this choice. Explicit information concerning the source location was missing at the time the prediction was made, and a location of N21,W08 was assumed on the basis of the NOAA/SEC forecaster's best estimate. This location was later confirmed on the basis of the SOHO/EIT observations mentioned above (Thompson et al., 1998).

In the present study, the HAFv. 2 model was rerun several times to obtain a "best fit" match between the predicted and measured shock arrival times at L1. This match was obtained when the shock velocity was close to $500 \mathrm{~km} / \mathrm{s}$. Figure 3 shows a sequence of eight snapshots from a continuous HAFv. 2 simulation obtained for this "best match" case (the conventions of presentation are the same as those described above in Sect. 4.1). At some longitudes the IMF lines appear to change color. This is an artifact resulting from taking an ecliptic plane slice through a wavy current sheet. Starting on 12 May 1997 from about 00:00 UT, compressed regions of spiral IMF indicate that four CIRs, viewed from above the Sun's North Pole, were already established in the heliosphere. In subsequent frames, these CIRs are seen to rotate counter clockwise. In Frame 4, a CIR overtakes the Earth on 13 May at about 12:00 UT. The near-Earth interplanetary region is characterized at that time by being in a "toward" IMF sector, showing enhanced solar wind velocity, density and IMF magnitude. In this view, the leading edge of the interplanetary shock has advanced to about $0.3 \mathrm{AU}$, with the center of the shock envelope directed along the Sun-Earth line. In Frames 5 and 6, the shock continues to advance along a line directed towards the Earth. The Shock-Earth connected lines map to a longitude well to the west of the 


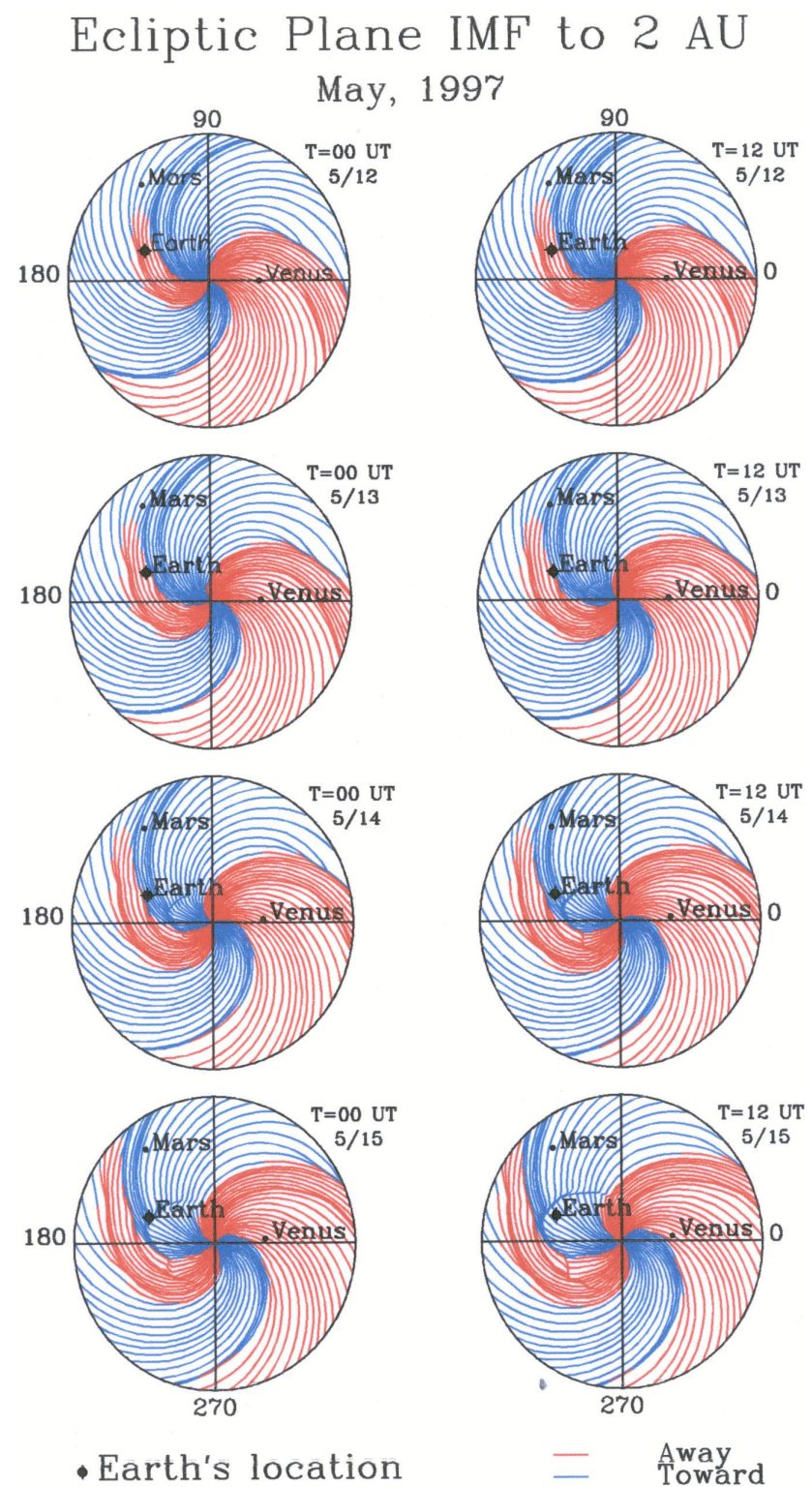

Fig. 3. Ecliptic plane simulation by the HAFv. 2 model of the temporal IMF disturbance caused by the shock from Event 1 (initiated on 12 May 1997). Polarity is indicated by blue (toward the Sun) and by red (away). The location of the Earth is indicated by a large black dot; of Venus and Mars, by smaller dots.

Central Meridian (CM). The temporally changing point of connection to the shock of these IMF lines is called the COB point (Connection with OBserver point), defined by Lario et al. (1998).

In Frame 7, which corresponds to 15 May at 00:00 UT, the simulated shock is seen to be just about to intersect with the Earth, thereby agreeing $(\Delta \mathrm{Th}=-1 \mathrm{~h})$ with the data recorded at both WIND and SOHO. This ex post facto result is a considerable improvement on the "real-time" result $\Delta \mathrm{Th}=$ $-31 \mathrm{~h}$ given in Table 2, Column 17. Also, the Earth connected IMF lines are shown in Frame 7 to map back to the Sun at western longitudes that are much nearer to the Sun-
Earth line than was the case in earlier frames.

On 15 May at 12:00 UT (last frame), the leading edge of the modeled shock has propagated beyond the Earth's orbit. Its envelope is distorted because the shock is seen to have advanced faster on its western flank (when compared with its motion along the CM line) as it rode over regions of higher speed ambient solar wind streams.

When the ISPM and STOA models were also rerun with an initial shock velocity of $500 \mathrm{~km} / \mathrm{s}$, they yielded unsatisfactory results. For example, the difference between the predicted and observed arrival times was $+16 \mathrm{~h}$ in the case of ISPM and $+25 \mathrm{~h}$ in the case of STOA. Improved values of $8 \mathrm{~h}$ and $+10 \mathrm{~h}$ were correspondingly obtained when a shock velocity of $700 \mathrm{~km} / \mathrm{s}$ was adopted instead of $500 \mathrm{~km} / \mathrm{s}$.

The iterative ex post facto analysis outlined suggests that the coronal shock speed of a Central Meridian flare might reasonably be taken, when making real-time predictions, to be about $2 \times V_{\text {cme }}$ when the POS speed is lower than $\sim 1500 \mathrm{~km} / \mathrm{s}$. This ad hoc procedure might also be followed when the metric Type II speeds are inordinately large and also when multiple, mutually inconsistent, reports are under consideration.

\subsection{Interacting shocks: shock events 4 and 5 (20 February 2000)}

Event 4: An M2/1B flare with GOES-8 X-ray maximum at 18:52 UT was recorded in AR 8869 at S25,W16 on 17 February 2000. Palahua reported the start of a metric Type II drift with $V_{s}=700 \mathrm{~km} / \mathrm{s}$ at this time. A halo CME with $V_{\mathrm{cme}}=550 \mathrm{~km} / \mathrm{s}$ was visible in SOHO/LASCO images from 20:06 UT (S. Plunkett, real-time private communication, 2000). An EIT wave was also reported in real time. $\tau$ was taken by the forecasters to be $0.66 \mathrm{~h}$.

Event 5: An M1/2N flare with GOES-8 X-ray maximum at 20:35 UT was recorded at S29, E07 in AR 8872. Culgoora reported the start of a metric Type II drift at 20:25 UT with an estimated coronal shock speed of $550 \mathrm{~km} / \mathrm{s}$. Another halo CME (see above) was visible in SOHO/LASCO images from 21:30 UT, as later reported by S. Yoshira (http: //lasco-www.nrl.mil/cmelist.html). This latter CME is inferred here to have merged with the preceding halo CME. The associated flare was accompanied by an EIT wave with related dimming (S. Plunkett, real-time private communication, 2000). $\tau$ was taken by the forecasters to be $1.17 \mathrm{~h}$ (Table 1, Column 8).

Events 4 and 5 were separated at the Sun by $<2 \mathrm{~h}$. It is noted that STOA and ISPM are not designed to handle such multiple events, although ISPM can signal that potentially interacting shocks are present in the data.

The shock resulting from Event 4 was faster than that of Event 5, although of a shorter driver duration. As the source of Event 4 was $16^{\circ}$ west of $\mathrm{CM}$ and that of Event 5 was approximately $20^{\circ}$ further to the east of this location, forwardreverse-shock interactions upstream of the Earth are unlikely to have played a role in determining the outcome at $1 \mathrm{AU}$. The STOA and ISPM models predicted the second shock to 
Ecliptic Plane IMF, Velocity, Density, and Dynamic Pressure to 2 AU
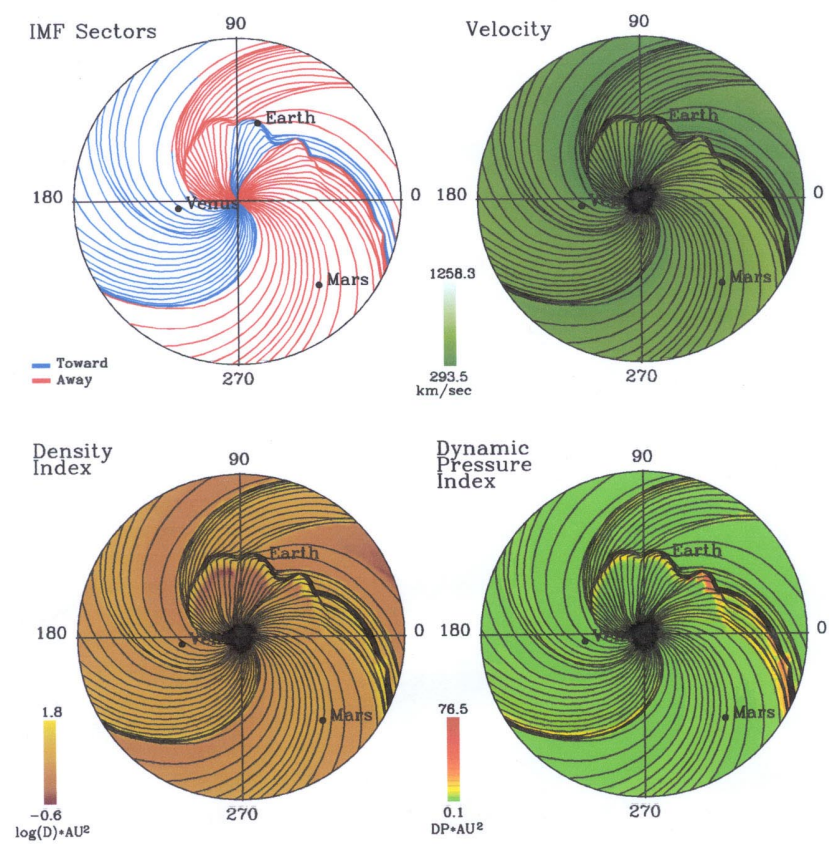

Feb 20, 2000

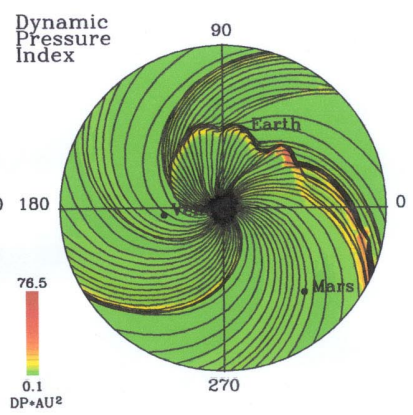

$\mathrm{TIME}=12 \mathrm{UT}$

Fig. 4. Ecliptic plane simulation of the IMF by the HAFv. 2 model showing the coalesced shocks from Events 4 and 5 at the time of impact with Earth. Upper left: IMF distortions at shock arrival on 20 February 2000. Upper right: spatial profile of solar wind velocity. Lower left: spatial profile of solar wind density. Lower right: spatial profile of solar wind dynamic pressure. The latter two physical parameters are normalized by $(\mathrm{AU})^{2}$, as shown by the color bars.

arrive several hours after the first (see Table 2). Since, however, the second shock was travelling through the high speed wake of the first, it can reasonably be expected to have caught up and interacted with the first shock close to $1 \mathrm{AU}$ to form a composite forward shock. The second event is classified here as "in" in the "predictions" Columns (15-16) for STOA and ISPM, where "in" stands for "interacting" (implying that a second discrete shock was not expected to associatively arrive). Event 5 is, in parallel, designated by "cn" for correct null (Sect. 4) in contingency Columns 18 and 19.

HAFv.2, which is able to process events that are closely spaced in time, predicted one shock resulting from the interaction of Events 4 and 5, with Shock 4 overtaken by Shock 5 . As was done above when using the STOA and ISPM models, Event 5 is classified as "in" in the predictions Column 17 of Table 2, and as "cn" in the associated contingency Column 20. The composite event can, meanwhile, be classified as a HIT (h) for Event 4 in contingency Column 20.

Details of the composite shock structure cannot be discerned due to the temporal and grid scale limitations of the simulation. However, it is clear that the simulated composite shock showed signification deformation at a scale of $1 \mathrm{AU}$.
Figure 4 presents four different ecliptic plane simulations produced by the HAFv. 2 model showing the coalesced shocks from Events 4 and 5 at their time of impact with the Earth. The four simulated parameters (IMF sectors, solar wind plasma velocity and normalized density and dynamic pressure indices) show the irregular shape of the shock as it interacts with the upstream, non-uniform flow and sector boundaries. The compressed solar wind directly behind the shock, followed by an extensive rarefaction zone, is clearly indicated, thus providing a simulated global insight into the temporal and spatial ecliptic plane dynamics.

\subsection{Interacting shocks: shock events 9 and 10 (23 January 2000)}

Event 9: An M1/SF flare at S07, E40 in AR 8813 on 20 January 2001 showed X-ray maximum in the GOES-8 record at 18:47 UT. Sagamore Hill detected a metric Type II drift from 18:42-18:53 UT with an estimated shock speed $V_{s}=$ $700 \mathrm{~km} / \mathrm{s}$. A halo CME was recorded in SOHO/LASCO images from 19:31 UT with $V_{\mathrm{cme}}=673 \mathrm{~km} / \mathrm{s}$. ACE $/ \mathrm{SWEPAM}$ measurements indicate that the solar wind speed $\left(V_{s w}\right)$ was $320 \mathrm{~km} / \mathrm{s}$. The forecasters estimated the piston driving time $\tau$ to be $0.67 \mathrm{~h}$.

Event 10: A more energetic M8/2B flare occurred in the same active region at S07, E46 with GOES-8 Xray maximum at 21:20 UT. A halo $\mathrm{CME}$ was recorded in SOHO/LASCO images from 21:54 UT with $V_{\text {cme }}=$ $1576 \mathrm{~km} / \mathrm{s}$. Holloman reported the occurrence of a metric Type II drift event from 21:14-21:48 UT with an estimated coronal shock speed $V_{s}=1300 \mathrm{~km} / \mathrm{s}$. The solar wind speed measured on ACE/SWEPAM was $V_{s w}=315 \mathrm{~km} / \mathrm{s}$. $\tau$ was taken to be $1 \mathrm{~h}$.

These solar events were separated by $\sim 2.5 \mathrm{~h}$. STOA predicted the second shock to arrive prior to the first one. ISPM predicted the first shock to decay to an MHD wave. Both solar sources were located at about $40^{\circ}$ east of Central Meridian, thus removing any concern that forward-reverse interactions upstream of $1 \mathrm{AU}$ associated with Event 9 would cancel, or substantially reduce, the effect of Shock Event 10. Therefore, (see Table 2) the second shock prediction can be taken to supercede the first. The prediction for Event 9 is described in Column 15 for STOA by the symbol "in" (for interacting) and in Column 16 for ISPM by "mhd" (for mhd wave). Event 9, as was done in Sect. 4.3, is classified as "cn" in the contingency Columns (18 and 19) for STOA and ISPM.

The HAFv. 2 results provide insight into the arrival of the composite shock at the Earth, as already shown for the previous case discussed in Sect. 4.3. Since a single shock is predicted from the interaction of Events 9 and 10, as was done before, Event 9 is classified as "in" in the predictions Column 17 and as "cn" in the contingency Column 20. The composite shock can be classified as a HIT (h) for Event 10. 


\section{Discussion}

Our discussion is concerned with the following important topics: Inherent problems in numerical modeling (5.1); Comparison of predicted with measured shock arrival times (5.2); Statistical analysis (5.3); Steps to improve model performance (5.4); Ejecta driven shocks and their accelerated particles (5.5); Energetic particle enhancements (5.6); Local planarity of shocks and their tilt relative to the Sun-Earth line (5.7).

\subsection{Inherent problems in numerical modeling}

Numerical modeling of interplanetary shocks propagating from the Sun to the Earth is fraught with uncertainties. Shock genesis close to the location of the parent flare is probably the safest assumption made. Significant uncertainties, meanwhile, exist in measuring the initial shock speed and in acquiring knowledge of those non-uniformities in the medium through which the shock passes. The seminal models considered here (STOA, ISPM and HAFv.2) are each physically based in fundamental theory with (a) some added empiricism in the case of STOA; (b) 2.5D MHD simulations in the case of ISPM and (c) the availability of solar surface magnetograms in the case of HAFv.2. The latter, very importantly, provides insights into the non-uniformity of the medium that is traversed by, and which influences the modeled effect of, a flare pulse. This non-uniformity of the medium affects the shock's curvature to a large extent, as suggested in Figs. 1, 3 and 4. Another potential contributor to the non-smoothness of the shock may be the irregular leading edge of the driving ejecta (driver), as suggested by Berdichevsky et al. (2000, 2002).

The plethora of coronal density models currently available, which are used in a variety of ways within the scientific community to determine shock velocities, are individually unsatisfactory. They are all spherically symmetrical. Also, they are empirically based on a large number of "quiet" Sun white light measurements. Estimates of individual coronal shock speeds depend, meanwhile, on the radial density profiles located above the flare site, that is on the profiles made at a given location at a specific time. The uncertainty that is inherent in accessing that very important parameter the metric Type II shock speed, is, consequently, dependent on the relative uncertainty in the coronal density gradient (considered to be about a factor of 2 at the time of writing). This situation begs the need for the development of a global 3-D model that can be used in real time to direct attention to the location of that solar event associated with the generation of a particular shock at a particular location and time.

Reliance on the parameter $\tau$ as a proxy for energy output (Sect. 3.1) is also unsatisfactory. The problem here is that there is no real-time capability to estimate the total mechanical energy of flare output. Only one flare to our knowledge has ever been scrutinized for both electromagnetic and mechanical energy output (Canfield et al., 1980; Webb et al., 1980).
It is noted that forecasting shock propagation in real time for non-flare CMEs is probably less accurate (albeit not attempted in this paper) than for flare CMEs, due to the lack of suitable "blast-off" diagnostics. While this pessimistic view may be true for disk events (helmet streamer blowouts), this input deficiency may be rectified by STEREO observations in the future.

5.2 Comparison of predicted with measured shock arrival times

Against the background of the problems discussed above, we may now consider the results of comparing the predicted with the measured shock arrival times. Table 2, Column 15 shows that, in the case of the STOA model, only one prediction (for Event 1) significantly approached the upper threshold of $24 \mathrm{~h}$ defined to allow a HIT (h) to be achieved. ISPM and HAFv. 2 each scored a MISS (m) in this case. The realtime predictions made for Event 1 (and also for Event 2) date back to 1997 when metric Type II burst information was less accurate than was afterwards the case, due to later upgrading of the hardware (digitization and bandwidth extension) at many of the recording sites. It is shown in Sect. 4.2 that, if a realistic value of the shock speed $(\sim 500 \mathrm{~km} / \mathrm{s})$ had been used for Event 1 rather than the value of $1400 \mathrm{~km} / \mathrm{s}$ originally adopted, HAFv. 2 would have scored a close HIT (to within $\Delta \mathrm{Th}=-1 \mathrm{~h}$ ). STOA and ISPM performed less well when the revised shock velocity was input, but improved $\Delta \mathrm{T}$ values of $8 \mathrm{~h}$ and $+10 \mathrm{~h}$ were achieved for an assumed coronal shock velocity of $700 \mathrm{~km} / \mathrm{s}$. This indicates the extreme sensitivity of the models to uncertainties in determining the coronal shock velocity.

It is recalled that Event 1 had the longest driver duration of all the events in the present sample $(2.5 \mathrm{~h})$. For STOA and ISPM a crude proxy for the input energy pulse is provided by the duration of the soft X-ray burst. This was truncated to $2 \mathrm{~h}$ in the case of ISPM (see Sect. 3.2).

There were two instances (Events $4 / 5$ and Events 9/10) when temporally close shock pairs at the Sun were inferred to produce a single composite shock at the Earth.

After 1997, STOA provided overall (for our sample of 11 events) $\Delta \mathrm{T}$ values in the close HIT range $1-8 \mathrm{~h}$. ISPM and HAFv. 2 also provided HITS for these events but with a broader margin of error.

\subsection{Statistical analysis}

Model performance in the present study, as indicated in Table 2 (Columns 18-20) on the basis of the Contingency Tables routinely employed in real-time Space Weather Forecasting, provide some confidence that the modeling philosophy and techniques used produce a satisfactory simulation of shock propagation in the Sun-Earth space. However, it is still necessary to address the question: can the models allow for a significantly better job to be made of predicting the arrival times of shock waves than can be derived from the measured transit velocity? 
To ascertain this statistically, the ratio of the calculated standard deviation of the transit times to Earth to the standard deviation of the measurements was estimated (treating interacting events as composite shocks) for each model, and these ratios turned out to be $0.60,1.15$ and 1.02 for STOA, ISPM and HAFv.2, respectively. It is recalled that, in Sects. 4.2 and 5.2, it is pointed out that in the case of Event 1, the large shock velocity $(1400 \mathrm{~km} / \mathrm{s})$ used in making the realtime prediction is not justified (i.e. this event constitutes a case where the Type II speed is not reliable). Inclusion of this event causes the standard deviations to associatively be quite large and this significantly influences the result. If Event 1 is omitted from the sample, the ratios become $0.36,0.76$ and 0.81 for STOA, ISPM and HAFv.2, respectively, suggesting that STOA performed significantly better than would a model with no predictive capability. While it can be argued that all events have their peculiarities, Event 1 is particularly problematical and unduly affects the statistical outcome of the small sample used for analysis.

Larger statistical samples should now be investigated. At the present time, a study using 36 cases comparing the performances of STOA, ISPM and HAFv.1, and another involving 173 cases comparing STOA, ISPM and HAF v.2, each in terms of the Contingency Model and skill scores only, is contained in Smith et al. (2000) and in Fry et al. (2001a, b).

\subsection{Steps to improve model performance}

As already indicated in Sect. 5.2, all the models showed extreme sensitivity to uncertainties in determining the initial coronal shock velocity. To overcome this problem, development of a global 3D MHD coronal density model temporally and spatially appropriate for specific events is recommended.

For further improved performance, HAFv. 2 should be upgraded to a full 3-D MHD solar wind model - when the required codes mature to a point where they can be run in real time using the available observational data inputs.

Uncertainties in the input data caused the setting by the forecasters of thresholds for detecting HITS and MISSES (Sect. 4) that allowed the shocks associated with those large, Earth directed, halo CMEs studied here, to be successfully predicted. Events of lower energy could have, in general, been missed. Statistical studies of relatively large samples are now required to provide guidance as to the criteria to be adopted when modeling the propagation of weaker shocks through the generally non-uniform interplanetary medium.

\subsection{Ejecta driven shocks and their accelerated particles}

Energetic particle records provide a medium complementary to solar wind plasma and interplanetary magnetic field data to validate shock arrival predictions. They have been used for the first time in the present study in this connection. In addition, energetic particle records are useful in that they potentially provide insights into the mechanisms of shock particle energization, as well as indicate the influence of shock strength on particle fluxes (topics not specifically considered in the present study). Further, they provide a tool for forecasting geomagnetic storms for individual events. A new study (Z. Smith, W. Murtagh and C. Smithtro, private communication, 2001) concerning 115 energetic particle enhancements measured aboard ACE indicates that, if a particular particle flux level is exceeded in the $47-65 \mathrm{keV}$ range, a geomagnetic storm with an above average $K_{p}$ index can be expected.

Modeling of the expected profiles of energetic proton events in the range $60 \mathrm{keV}$ to $100 \mathrm{MeV}$ by Lario et al. (1998) is based on the concept of the COB point (i.e. that point on the front of the shock that connects with the observer through the IMF lines). As the associated shock moves outwards from the Sun, the COBpoint moves along the shock front. This behavior is interpreted in the context of an MHD modeled, variable width, non-circular, decelerating parent shock in a background Archimedian configuration to appropriately infer the nature of the rise times of those individual particle profiles associated with shocks originating to the west, at Central Meridian and to the east of the Sun (as also discussed by Lario et al., 1998).

The difference between the shock speed pertaining to any time and at any position on its 3-D surface and the background solar wind speed, constitutes a source of critical inherent uncertainty. This difference determines the likely nonuniform geometry of the shock, i.e. its deviation from being quasi-spherical (cf. the review by Dryer, 1974). Thus, the position of the parent flare on the solar disk and the local directionality of the shock at the time of its formation, are each important factors in forecasting the arrival of a particular shock at the Earth and in making energetic particle flux predictions. We recall that the large-scale structure of interplanetary shocks was first explored using energetic particle observations (Sanahuja et al., 1983; Cane et al., 1988).

Also, it was observed and theoretically supported (see the review by Dryer, 1994) that initially fast shocks (say $>1500 \mathrm{~km} / \mathrm{s}$ ) decelerate rapidly, whereas initially slow shocks (say $<1000 \mathrm{~km} / \mathrm{s}$ ) decelerate very slowly. The latter are, thus, often mistakenly identified as shocks of constant velocity. Shocks in both of these "classes" can be characterized by either a reduction, or even in an increase, in speed depending on whether they transit coronal hole fast streams or pass through those slower streams emanating from above helmet-streamers. For example, it has recently been reported by Manoharan et al. (2001) on the basis of interplanetary scintillation measurements made at Ooty, India and Toyokawa, Japan that the ICME associated with the so-called "Bastille-Day shock" on 14 July 2000 (Event 8 in the present sample) slowed from $\sim 14 \mathrm{~ms}^{-2}$ at $R<100 R_{S}$ to $\sim 6 \mathrm{~ms}^{-2}$ at $R>100 R_{s}$.

The ratio of $V_{s h} / V_{t r}$ (Table 2, Column 7) obtained for each of the eleven events considered here, is in the range 0.7-0.9, for all but Shock Event 9. This result is in conformity both with observations and with MHD simulated studies of individual non-interacting events (cf. Watari and Detman, 1998; Smith et al., 1996). The higher ratio associated with Shock 9 implies that it arrived faster than is usual. This 
could be anticipated because this interplanetary shock was inferred to be the result of the interaction of two energetic solar shocks (Sect. 4.4). In the case of an earlier interacting pair, Events 4 and 5 (Sect. 4.3), the ratio remained within the expected range because these were shocks of comparable energy and, thus, not expected to interact until they were close to $1 \mathrm{AU}$. In these circumstances, their resultant would not have advanced significantly faster (see also Smith et al., 1986).

The subject of the behavior of ejecta-driven shocks beyond the expiration of the basically crude piston driving time $\tau$ used in the present models is also of interest. Lepping et al. (2001a, b) found that for Events 1, 3 and 8 the leading edge speeds of these three magnetic clouds appeared to be similar to the velocities of their associated precursor shocks. (It is not known if this equality is also a feature in the case of other types of ejecta.) The real-time success of the present models for Events 3 and 8 (we exclude from consideration here, see Sect. 4.2, the real-time predictions made in the case of Event 1) is interesting despite the lack of a modeled physical driving mechanism beyond the end of $\tau$. It is noted that the models tested (Sect. 3) included only kinetic energy inputs and did not take into account the internal magnetic energy associated with the flux tubes known to be present in magnetic clouds. Other forms of input pulses (for example, momentum, thermal energy, etc.) could, of course, be potentially more easily mimicked for initialization purposes. In any case, this very interesting topic of the indicated predictive success of, as yet, rather simple models must be considered to pose an open question.

\subsection{Energetic particle enhancements}

HAFv. 2 is particularly useful in that it provides, based on its solar input parameters, information on the non-uniform conditions in the heliosphere through which shocks propagate, while also monitoring how the COBpoint (which influences particle rise times for temporal flux enhancements) changes with time. Further, it simulates the development of shock deformations on a scale of fractions of an AU. Such deformations can result from the influence of upstream structural inhomogeneities. In addition, an influence may be exerted due to variations in the spatial profile of the driver ejecta.

HAFv. 2 allows an improvement to be made in the interpretation of energetic particle flux profiles based on simpler spherical treatments of the shock front (see, e.g. Ng et al., 1999), because superimposed on the measured particle energy enhancement profiles is the influence of interplanetary structures in modulating the trajectories followed by particular particles. Figure 2 shows the particle record associated with a flare (Event 1) that was generated at N21,W08 (Central Meridian). Figure 3 shows the simulated behavior of the COBpoint during this event as it mapped back to the Sun at western longitudes. The realistic shock shape simulated by HAFv.2 (rather than a purely spherical or cylindrical shape) is particularly useful in studying such particle profiles.
5.7 Local planarity of shocks and their tilt relative to the Sun-Earth line

Signatures showing the shock arrival times of the eleven events in our sample were recorded aboard several spacecraft between L1 and the Earth in energetic particle, IMF and plasma data. An overview of these data is provided in Table 3.

Some of the energetic particle enhancements listed were recorded aboard SOHO by the LION and EPHIN instruments within the composite energy range $\sim 40 \mathrm{keV}-50 \mathrm{MeV}$ (McKenna-Lawlor et al., 1997; Muller-Mellin et al., 1995). Also, proton data were recorded by the DOK-2 instrument on INTERBALL-1 in the range $26 \mathrm{keV}-600 \mathrm{keV}$ (Lutsenko et al., 1998). Aboard ACE, contemporaneous data were recorded by the Solar Wind Experiment Proton Alpha Monitor (SWEPAM) and by the magnetometer (MAG) described by Zwickl et al. (1998). In addition, energetic proton data were recorded aboard IMP-8 by the Energetic Proton Experiment EPE (Williams, 1977) in the range $50 \mathrm{keV}$ to $25 \mathrm{MeV}$ and by the Charged Particle Measurements Experiment CPME (Armstrong et al., 1978) in the range 0.29$500 \mathrm{MeV}$.

Details concerning the complementary IMP-8 plasma and magnetic field experiments used to detect shock arrival times are given, respectively, by Richardson et al. (1994) and by Mish and Lepping (1976). Magnetic field data from this spacecraft were not available after 16 June 2000. NASA's International Solar Terrestrial Physics (ISTP) products and data are described by Mish et al. (1995). Solar wind plasma data from INTERBALL-1 were obtained from the VDP instrument (Safrankova et al., 1997). The plasma, magnetic field and energetic particle instruments aboard WIND are described by Ogilvie et al. (1995), Lepping et al. (1995) and von Rosenvinge et al. (1995), respectively.

In addition to the spacecraft data, Table 3, Column 14 provides the individual times of shock arrival at the Earth itself, as indicated by an SSC. These data show that, correcting for the two pairs of interacting events (Sects. 4.3 and 4.4.), all of the large, halo related particle events investigated were followed by an SSC that, in turn, was followed by a significant $K_{p}$ index (Columns 15-17). It is noted that we do not consider $B_{z}$ here. Also, we do not imply that the shocks produced these $K_{p}$ indices.

Close temporal trends are visible in the available shock arrival times. In particular, Table 3, Columns 3-5 and 1214 reflect shock advancement from one spacecraft to another while en route from L1 to the Earth (for brevity, not all of the spacecraft positions are listed in the Table).

Figures 1 and 3 (Frames 3-5) and Fig. 4 (upper left Frame) show that, for shock Events 7,1 and 9/10 on a scale of several hundred Earth radii (i.e. within the compass of the black dot representing the Earth), the shock events can be considered to be locally planar (not spherical) on a $300 R_{E}$ spatial scale. An assumption concerning the local planarity of shocks used (Berdichevsky et al. 2000) in a study of the profiles of 42 shocks that included Event 1 (15 May 1997) in the 
Table 3. Shock arrival times at various spacecraft near the Earth

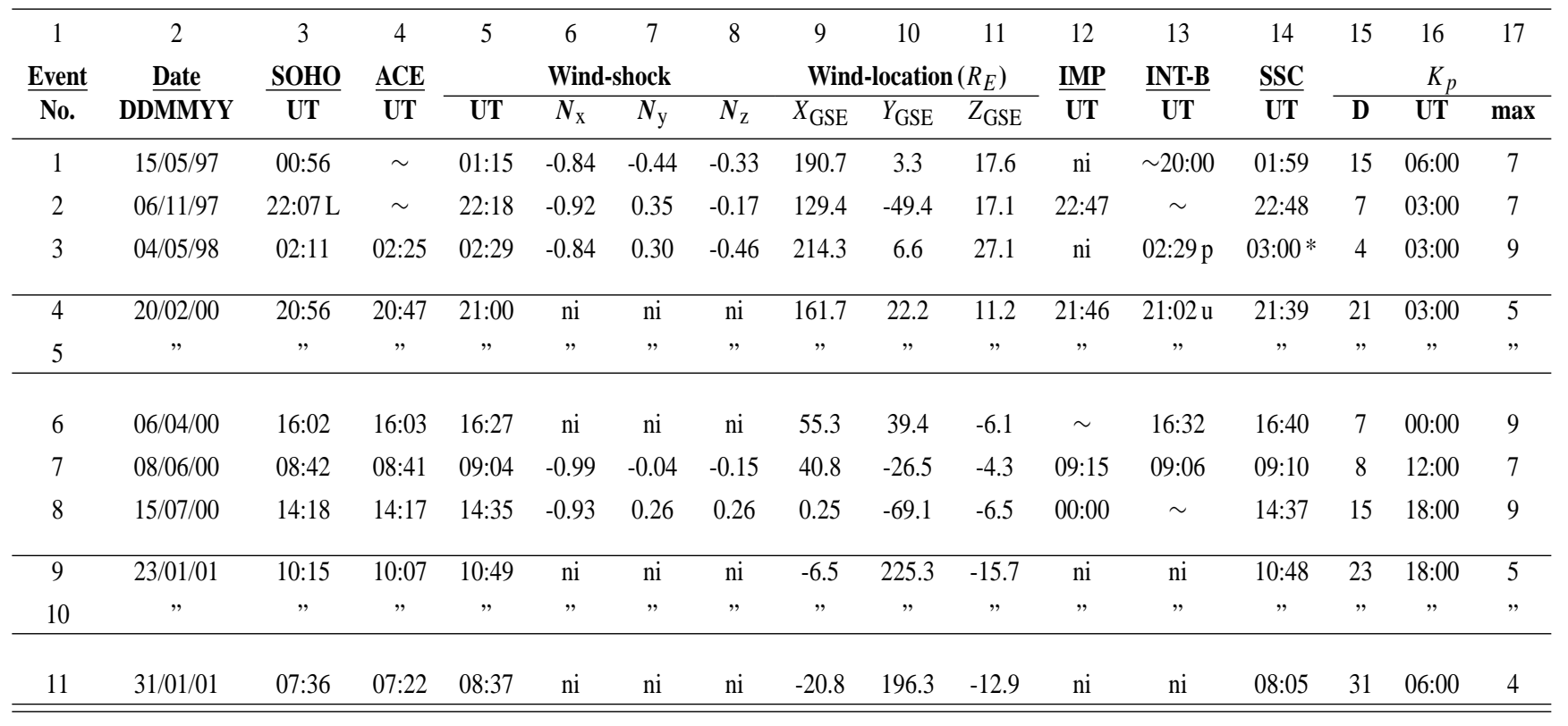

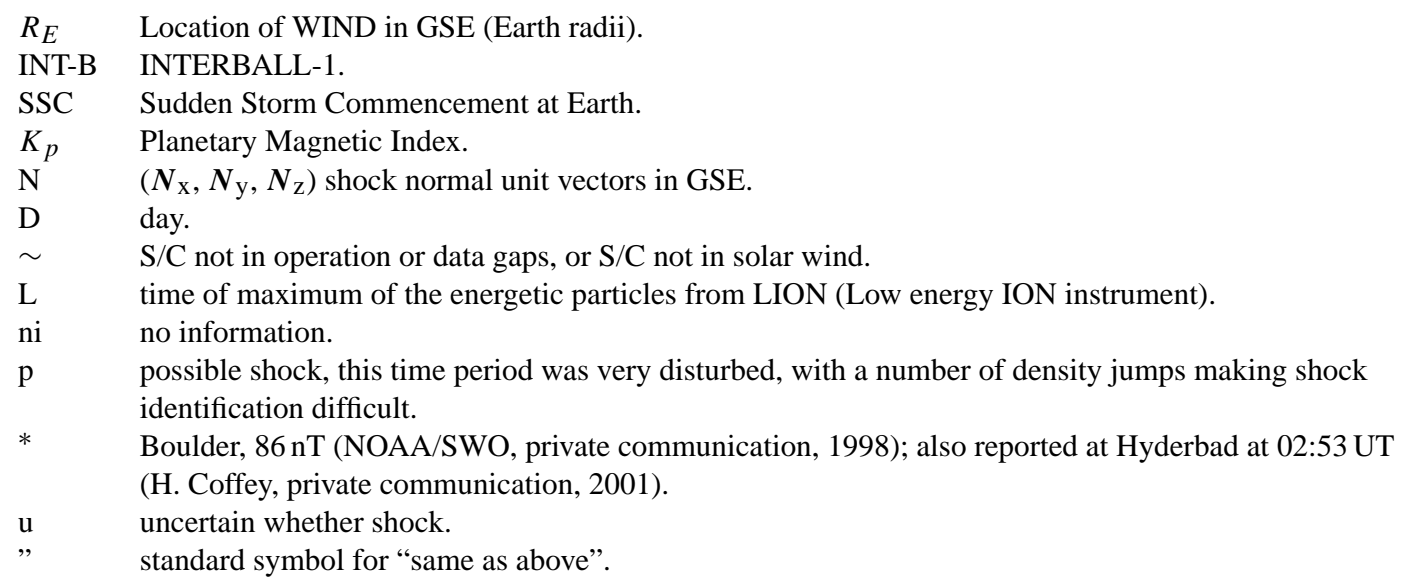

Columns 1-2: $\quad$ Event numbers and arrival dates of shocks at near-Earth spacecraft.

Cols. 3-5, 12, 13: $\quad$ Shock arrivals at SOHO, ACE, WIND, IMP-8 and INTERBALL-1.

Cols. 6-8: $\quad$ Shock normal unit vectors in GSE at WIND.

Cols. 9-11: $\quad$ Location of WIND in GSE (components in Earth radii).

Column 14: Sudden Storm Commencement at Earth.

Cols. 15-17: $\quad$ Day, time of start and maximum value of $K_{p}$.

present sample, can be supported over a range of hundreds of Earth radii (A. Szabo, private communication 2001). A similar result was found to hold for shock Event 8 (15 July 2000) which was discussed by Lepping et al. (2001a). In a multi-point study (Berdichevsky et al., 2001) it was shown that the observations were consistent with the interpretation that every spacecraft sensed a similar value of the shock normal vector for all the events considered, including Event 3 in our sample (4 May 1998). The spacecraft concerned were all situated at locations within 300 Earth radii (i.e. they were distributed over a relatively small spatial scale).

It is now of interest to check the direction of shock propagation versus the normal direction measured on WIND. In principle, assuming that the solar wind speed is parallel to the $x$-axis, one can simply calculate the shock normal vector from measurements made at three different positions in the following way; based on the value of the solar wind velocity one can determine the locations of the spacecraft considered at a time when the shock hits one spacecraft, then fit a plane to the three points, which will be the shock plane. In practice, however, it turned out that all the spacecraft were relatively close to the $x-y$ plane, i.e. the differences in the $z$ components of their positions were small compared with their $x$ and $y$ differences. Therefore, such a procedure yielded normal vectors with unreasonably large $z$ components.

While keeping in mind that the determination of the local 
Table 4. Comparison of the components of the shock normal vectors detected at Wind with those determined using a two point method at SOHO/Wind and ACE/Wind

\begin{tabular}{|c|c|c|c|c|c|c|c|}
\hline \multirow{3}{*}{$\begin{array}{c}1 \\
\text { Event } \\
\text { No. }\end{array}$} & \multirow{3}{*}{$\begin{array}{c}2 \\
\underline{\text { Date }} \\
\text { DDMMYY }\end{array}$} & 3 & 4 & 5 & 6 & 7 & 8 \\
\hline & & \multicolumn{3}{|c|}{ WIND-shock normal } & \multicolumn{3}{|c|}{ SOHO/WIND shock normal } \\
\hline & & $N_{\mathrm{X}}$ & $N_{\mathrm{y}}$ & $N_{\mathrm{Z}}$ & $\boldsymbol{n}_{\mathrm{X}}$ & $n_{\mathrm{y}}$ & $n_{\mathrm{Z}}$ \\
\hline 1 & $15 / 05 / 97$ & -0.84 & -0.44 & -0.33 & -0.82 & -0.27 & -0.5 \\
\hline 2 & 06/11/97 & -0.92 & 0.35 & -0.17 & -0.88 & 0.47 & -0.24 \\
\hline 3 & 04/05/98 & -0.84 & 0.3 & -0.46 & -0.66 & 0.27 & $-0.75^{*}$ \\
\hline $4 / 5$ & $20 / 02 / 00$ & ni & ni & ni & -0.985 & 0.2 & 0.08 \\
\hline 6 & 06/04/00 & ni & ni & ni & -0.87 & -0.48 & -0.15 \\
\hline 7 & 08/06/00 & -0.99 & -0.04 & -0.15 & -0.965 & -0.26 & -0.09 \\
\hline 8 & $15 / 07 / 00$ & -0.93 & 0.26 & 0.26 & -0.84 & 0.5 & $0.21 *$ \\
\hline $9 / 10$ & $23 / 01 / 01$ & ni & ni & ni & -1 & -0.009 & 0.32 \\
\hline 11 & $31 / 01 / 01$ & ni & ni & ni & -0.97 & 0.25 & 0.16 \\
\hline
\end{tabular}

* $\quad$ ACE data used instead of SOHO data (more favorable relative position)

Event 5 interacts with Event 4, hence treated as a single event. ni no information

Event 10 interacts with Event 9, hence treated as a single event.

solar wind shock velocity $V_{s h}$ has a relatively large error, the $x$ and $y$ components of the shock normals can alternatively be determined from the $x$ and $y$ positions of two spacecraft in the $x-y$ plane, assuming that their $z$ components are 0 . If it is then assumed that the projection of the "real" normal vector onto the $x-y$ plane is correct and coincides with the value just obtained, then the $n_{z}$ component can be determined by comparing the differences $\left(x_{1}-x_{2}\right)$ with the differences $\left(z_{1}-z_{2}\right)$. This procedure was used to calculate shock normal vectors for the events in our sample using data from SOHO and ACE (which always remained close to L1), and the values obtained were found to be relatively close to those obtained using shock data recorded at a single spacecraft (WIND) following a method described by Berdichevsky et al. (2001). (See Table 4 where the components of the normal vectors determined using these two methods are compared.) As noted above, the 3-point method generally yielded quite different normal vectors.

In Table 4, shock normal unit vectors calculated using plasma and magnetic field measurements made aboard WIND (Columns 3-5) can be compared with corresponding vectors computed from the measurements aboard SOHO/WIND and ACE/WIND (Columns 6-8). These shocks turned out to be non-radial (or tilted) relative to the Sun-Earth line, probably reflecting inherent directionality associated with their solar origin. It is noted that Fig. 4, in particular, based on HAFv.2, predicts the angle of the shock normal to be at about $45^{\circ}$ from the Sun-Earth line on 20 February 2000 . On the local spatial scale covered by the various upstream spacecraft interrogated, evidence in the data of local shock deformation is not expected.

\section{Conclusions}

Against a background of inherent practical problems, realtime predictions of shock arrivals at the Earth, generated by eleven halo CME related events, provided by three numerical models (STOA, ISPM and HAFv.2), were tested using plasma, IMF and energetic particle measurements. The results and conclusions are summarized as follows:

- The models were all generally successful in predicting shock arrivals. STOA scored the smallest values of "predicted minus observed" arrival times (typical precision better than $8 \mathrm{~h}$ ).

- The ratio of the calculated standard deviation of the transit times to Earth to the standard deviation of the measurements was estimated (treating interacting events as composite shocks) for each model and these ratios turned out to be $0.60,1.15$ and 1.02 for STOA, ISPM and HAFv.2, respectively. If an event for which the shock velocity was not well known is omitted from the sample considered, these figures become $0.36,0.76$ and 0.81 , respectively. Larger statistical samples should now be tested.

- Improvements in the predictive capability can be achieved through

(a) Development of a global 3D MHD coronal density model for use in estimating coronal shock speeds for specific events;

(b) Upgrading HAFv.2 to a full 3D MHD solar wind model;

(c) Making statistical studies of relatively large samples to obtain, in particular, guidance as to the 
criteria to be adopted when modeling the propagation of relatively weak shocks through the generally non-uniform interplanetary medium.

- Energetic particle records provide a medium complementary to solar wind plasma and IMF data to validate shock arrival predictions.

- HAFv.2 provides insight concerning how particle rise times can be influenced at the location of a spacecraft observer by the COBpoint for shocks emanating from particular solar locations.

- The ratio of the in situ shock velocity and the "Sun to L1" transit velocity $\left(V_{s h} / V_{t r}\right)$ was in the range $0.7-0.9$ for non-interacting events in the present sample.

- Shock deformation on a scale of the order of a fraction of an AU, hence transit times to specific heliospheric locations modeled by HAFv.2, can be influenced by upstream inhomogeneity in the solar wind, and possibly also by the uneven profile of driving ejecta.

- On the small spatial scale $\left(300 R_{E}\right)$ where near-Earth spacecraft are located (including L1), shocks are predicted by HAFv.2 to be locally planar. Measurements made at SOHO, ACE and WIND confirm that the passing shocks were planar close to the Earth. The shocks were also tilted relative to the Sun-Earth line, probably reflecting the inherent directionality associated with their solar origin.

Acknowledgements. SMcKL expresses appreciation of the financial support of the Office for Science and Technology, Ireland. K. K provides his thanks for Hungarian National Grant OTKA-034566. $\mathrm{K}$. $\mathrm{Ku}$ acknowledges the provision of support by the Slovak grant agency of VEGA \# 1147. Several of the authors (CDF, CSD, MD, WS) acknowledge support of the US DoD University Partnering for Operational Support (UPOS) Project A83-05,Geoeffectiveness of Solar Events. M D also thanks NOAA's Space Environment Center for their hospitality; he also thanks S. Plunkett, K. Schenk and the SOHO/LASCO and EIT operations group for their near realtime information on CMEs. DB also acknowledges support from NASA/ISTP and Grant NAG 5-10883. Data for the CME catalog is generated and maintained by the Center for Solar Physics and Space Weather at the Catholic University of America in cooperation with the Naval Research Laboratory and NASA. The modeled background solar wind flow and the IMF topology used in HAFv.2 are derived from synoptic solar source surface maps of the magnetic field and solar wind velocity close to the Sun. These data are updated and made available on a daily basis on the NOAA Space Operations website: http://www.sec.noaa.gov. Finally, we express our thanks to the two referees for their constructive suggestions.

The Editor in chief thanks M. Heinemann and M. Poquerusse for their help in evaluating this paper.

\section{References}

Akasofu, S.-I. and Fry, C. D.: A first generation geomagnetic-storm prediction scheme, Planet, Space Sci., 34, 77-92, 1986.
Andrews, M. D. and Howard, R. A.: A two-type classification of LASCO coronal mass ejections, Space Sci. Rev., 95, 147-163, 2001.

Arge, C. N. and Pizzo, V. I.: Improvement in the prediction of solar wind conditions using near-real time solar magnetic field updates, J. Geophys. Res., 105, 10 465-10 479, 2000.

Armstrong, T. P., Krimigis, S. M., and Lepping, R. P.: Magnetosheath bursts of predominantly medium nuclei observed with Imp 8 on February 16, 1974, J. Geophys. Res., 83, 5198-5206, 1978.

Berdichevsky, D., Szabo, A., Lepping, R. P., Vinas, A. F., and Mariani, F.: Interplanetary fast shocks and associated drivers observed through the twenty-third solar minimum by WIND over its first 2.5 years, J. Geophys. Res., 105, 27 289-27 314, 2000.

Berdichevsky, D., Lepping, R. P., Szabo, A., and Lazarus, A. J.: Estimation of interplanetary shock-normals using multispacecraft observations: Cases of shocks within minutes of magnetohydrodynamic discontinuities, EOS Trans. AGU, 82(20), Abstract SH42B-10, 2001

Berdichevsky, D. B., Farrugia, C. J., Thompson, B. J., Lepping, R. P., Reames, D. V., Kaiser, M. L., Steinberg, J. T., Plunkett, S. P., and Michels, D. J.: Halo-coronal mass ejections near the 23rd solar minimum: Lift-off, inner heliosphere, and in situ (1 AU) signatures, Ann. Geophysicae, this issue, 2002.

Brueckner, G. E., Howard, R. A., Koomen, M. J., Korendyke, C. M., Michels, D. J., Moses, J. D., Socker, D. G., Dere, K. P., Lamy, P. L., Llebaria, A., Bout, M. V., Schwenn, R., Simnett, G. M., Bedford, D. K. and Eyles, C. J.: The Large Angle Spectroscopic Coronagraph (LASCO), Solar Phys, 162, 357-402, 1995.

Cane, H. V., Reames, D. V., and Von Rosenvinge, T. T.: The role of interplanetary shocks in the longitude distribution of solar energetic particles, J. Geophys. Res., 93, 9555-9567, 1988.

Canfield, R. C., Cheng, C.-C., Dere, K. D., Dulk, G. A., McLean, D. J., Robinson, Jr., R. D., Schmahl, E. J., and Schoolman, S. A.: Radiative energy output of the 5 September, 1973 flare, in: Solar Flares, A Monograph from SKYLAB Solar Workshop II (Appendix A), (Ed) Sturrock, P. S., Publ. Colorado Associated University Press, Boulder, Colorado, USA, 1980

Desai, M. I., Mason, G. M., Dwyer, J. R., Mazur, J. E., Smith, C. W., and Skoug, R. M.: Acceleration of ${ }^{3} \mathrm{He}$ Nuclei at Interplanetary Shocks, Astrophys. J., 553, L89-L92, 2001.

Dryer, M.: Interplanetary shock waves generated by solar flares, Space Sci. Rev., 15, 403-468, 1974.

Dryer, M.: Interplanetary studies: Propagation of disturbances between the Sun and the magnetosphere, Space Sci. Rev., 67 (3/4), 363-419, 1994.

Dryer, M.: Comments on the origins of coronal mass ejections, Solar Phys., 169, 421-429, 1996.

Dryer, M. and Smart, D. F.: Dynamical models of coronal transients and interplanetary disturbances, Adv. Space Res., 4, 291-301, 1984.

Fry, C. D., Sun, W., Deehr, C. S., Dryer, M., Smith, Z., Akasofu, S.I., Tokumaru, M., and Kojima, M.: Improvements to the HAF solar wind model for space weather predictions, J. Geophys. Res., 106, 20 985-21 001, 2001a.

Fry, C. D., Dryer, M., Sun, W., Smith, Z., Deehr, C. S., and Akasofu, S.-I.: Forecasting Solar Wind Structures and Shock Arrival Times Using an Ensemble of Models using an Ensemble of Models, J. Geophys. Res., (submitted), 2001b.

Gonzalez, W. D., Joselyn, J. A., Kamide, Y., Kroehl, H. W., Rostoker, G., Tsurutani, B. T., and Vasyliunas, V. M.: What is a Geomagnetic Storm?, J. Geophys. Res., 99, 5771-5792, 1994. 
Gonzalez, W. D., Tsurutani, B. T., and Clua de Gonzalez, A.: Interplanetary origin of geomagnetic storms, Space Sci. Rev. 88, 529-562, 1999.

Gosling, J. T.: The Solar Flare Myth, J. Geophys. Res. 98, $18937-$ $18949,1993$.

Gosling, J. T. and Hundhausen, A. J.: Reply to Svestka, Solar Phys., 160, 57-60, 1995.

Kahler, S. W.: Solar Flares and Mass Ejections, Ann. Rev. Astron. Astrophys. 30, 113-141, 1992.

Kahler, S. W., Sheeley, Jr., N. R., Howard, R. A., Koomen, M. J., Michels, D. J., McGuire, R. E., Von Rosenvinge, T. T., and Reames, D. V.: Associations between coronal mass ejections and solar energetic proton events, J. Geophys. Res., 89, 9683-9693, 1984

Lario, D., Sanahuja, B., and Heras, A. M.: Energetic particle events: Efficiency of interplanetary shocks as $50 \mathrm{keV}<\mathrm{E}<100 \mathrm{MeV}$ proton accelerators, Astrophys. J., 509, 415-434, 1998.

Lepping, R. P. and Chao, J.-K.: A shock surface geometry, the February 15-16, 1987 event, J. Geophys. Res., 81, 60-64, 1976.

Lepping, R. P., Acuna, M. H., Burlaga, L. F., Farrell, W. M., Slavin, J. A., Schatten, K. H., Mariani, F., Ness, N. F., Neubauer, F. M., Whang, Y. C., Byrnes, J. B., Kennon, R. S., Ranetta, P. V., Scheifele, J., and Worley, E. M.: The Wind magnetic field investigation, Space Sci. Rev., 71, 207-229, 1995.

Lepping, R. P., Berdichevsky, D., Burlaga, L. F., Lazarus, A. J., Kasper, J., Desch, M. D., Wu, C.-C., Reames, D. V., Singer, H. J., Smith, C. W., and Ackerson, K. I.: The Bastille Day magnetic clouds and upstream shocks: Near-Earth interplanetary observations, Solar Phys., in press, 2001a.

Lepping, R. P., Berdichevsky, D., Szabo, A., Lazarus, A. J., and Thompson, B. J.: Upstream shocks and interplanetary magnetic cloud speed and expansion: Sun, Wind, and Earth Observations, in: COSPAR 2000, (Ed) Chao, J. K., Adv. Space Res., in press, $2001 b$.

Lewis, D. and Dryer, M.: Shock-Time-of Arrival Model (STOA87), NOAA/SEL Contract Report (Systems Documentation to USAF Air Weather Service), 1987.

Lutsenko, V. N., Kudela, K., and Sarris, E. T.:, The DOK-2 experiment to study energetic particles by the Tail probe and Auroral probe satellites in the INTERBALL project, Cosmic Res., 36 (1), 94-103, 1998.

Manoharan, P. K., Tokumaru, M., Pick, M., Subramanian, P., Ipavich, F. M., Schenk, K., Kaiser, M. L., Lepping, R. P. and Vourlidas, A.: Coronal mass ejection of July 14, 2000 flare event: Imaging from near-Sun to near-Earth environment, Astrophys. J., 559, in press, 2001.

McKenna-Lawlor, S. M. P., Elendt, I., Rusznyak, P., Kunow, H., Muller-Mellin, R., and Witte, M.: The LION instrument on SOHO and its scientific objectives, Ann. Geophysicae, 15, 1-4, 1997.

Mish, W. H. and Lepping, R. P.: Magnetic field experiment data processing system: Explorers 47 and 50, NASA-GSFC Report X-694-76-158, Greenbelt, MD, August 1976.

Mish, W. H., Green, J. L., Reph, M. G., and Peredo, M.: ISTP science data systems and products, Space Sci. Rev., 71, 815-878, 1995

Muller-Mellin, R., Kunow, H., Fleissner, V., Pehlke, E., Rode, E., Roschmann, N., Scharmberg, C., Sierks, H., Rusznyak, P., McKenna-Lawlor, S., Elendt, I., Sequeiros, J., Meziat, D., Sanchez, S., Del Peral, L., Witte, M., Marsden, R., and Henrion, J.: COSTEP - Comprehensive Suprathermal and Energetic Particle Analyser, reprinted from Solar Phys. 162, Nos. 1-2, 1995 in: "The SOHO Mission", (Eds) Fleck, B., Domingo, V., and Poland, A. I., Publ. Kluwer Academic Publishers, Dordrecht, Boston, London, 483-504, 1995.

Newkirk, Jr., G.: Solar corona in active regions and the thermal origin of the slowly varying component of solar radiation, Astrophys. J., 133, 983-1013, 1961.

Ng, C. K., Reames, D. V., and Tylka, A. J.: Effect of protonamplified waves on the evolution of solar energetic particle composition in gradual events, Geophys. Res. Lett., 26 (14), 2145 2148, 1999.

Odstrcil, D. and Pizzo, V. J.: Distortion of the interplanetary magnetic field by three-dimensional propagation of coronal mass ejections in a structured solar wind, J. Geophys. Res., 28, 28 225-28 239, 1999.

Odstrcil, D., Dryer, M., and Smith, Z.: Propagation of an interplanetary shock along the heliospheric current sheet, J. Geophys. Res., 101, 19973-19986, 1996.

Ogilvie, K. W.: SWE, Comprehensive plasma instrument for the Wind spacecraft, Space Sci. Rev., 71, 55-77, 1995.

Popecki, M. A., Desai, M. I., Skoug, R. M., Smith, C. W., Moebius, E., Galvin, A. B., Kistler, L. M., Klecker, B., and Zurbuchen, T. H.: SEP Fe Charge States in ${ }^{3} \mathrm{He}$-Rich Interplanetary Shock Events, Eos Trans. AGU, 82 (47), Fall Meeting Suppl., Abstract SH12C-03, 2001

Reames, D. V.: Acceleration of energetic particles by shock waves from large solar flares, Astrophys. J. (Lett), 358, L63-L69, 1990.

Reames, D. V.: Solar energetic particles: a paradigm shift, Rev. Geophys. (Suppl) 33, (U.S. National Report to the IUGG), 585589, 1995.

Reames, D. V.: Energetic particles and the structure of coronal mass, ejections, in: "Coronal Mass Ejections", (Eds) Crooker, N., Joselyn, A., and Feynman, J., Geophys. Monograph 99 (AGU), 217-226, 1997.

Reames, D. V.: Particle acceleration at the sun and in the heliosphere, Space Sci. Rev., 90, 413-491, 1999.

Reames, D. V. and Ng, C. K.: Streaming limited intensities of solar energetic particles, Astrophys. J., 504, 1002-1005, 1996.

Richardson, J. D., Paularena, K. I., Belcher, J. W., and Lazarus, A. J.: Solar wind oscillations with a 1.3 year period, Geophys. Res. Lett., 21, 1559-1560, 1994.

Roth, I. and Temerin, M.: Enrichment of ${ }^{3} \mathrm{He}$ and heavy ions in impulsive solar flares, Astrophys. J., 477, 940-957, 1997.

Safrankova, J., Zastenker, G., Nemecek, Z., Fedorov, A., Simersky, M., and Prech, L.: Small-scale observation of magnetopause motion: preliminary results of the INTERBALL project, Ann. Geophysicae, 15, 5, 562-569, 1997.

Sanahuja, B., Domingo, V., Wenzel, K.-P., Joselyn, J. A., and Kepler, E.: A large proton event associated with solar filament activity, Solar Phys., 84, 321-337, 1983.

Schaefer, J. T.: Critical Success index as an indication of warning skill, Weather and Forecasting, 3, 570-575, 1990.

Smart, D. F. and Shea, M. A.: A simplified model for timing the arrival of solar-flare initiated shocks, J. Geophys. Res., 90, 183190, 1985

Smart, D. F., Shea, M. A., Barron, W. R., and Dryer, M.: A simplified technique for estimating the arrival time of solar flare initiated shocks, Proc. of STIP Workshop on Solar/Interplanetary Intervals, in: Maynooth Ireland 4-6 August 1982, (Eds) Shea, M. A., Smart, D. F., and McKenna-Lawlor, S., Publ. Book Crafters Inc. Chelsea, MI, 139-156, 1984.

Smart, D. F., Shea, M. A., Dryer, M., Quintana, A., Gentile, L. C. and Bathurst, A. A.: Estimating the arrival time of solar flare- 
initiated shocks by considering them to be blast waves riding over the solar wind, in: Proc. Symp. on Solar-Terrestrial Predictions, (Eds) Simon, P., Heckman, G. R., and Shea, M. A., Meudon, France 18-22 June 1984, Publ. U.S. Government Printing Office, Washington, D. C., 471-481, 1986.

Smith, Z. and Dryer, M.: MHD study of temporal and spatial evolution of simulated interplanetary shocks in the ecliptic plane within 1 AU, Solar Phys., 129, 387-405, 1990.

Smith, Z. and Dryer, M.: The Interplanetary Shock Propagation Model: A Model for Predicting Solar-Flare-Caused, Geomagnetic Sudden Impulses Based on the 21/2D, MHD Numerical Simulation Results for the Interplanetary Global Model (2D IGM), NOAA Technical Memorandum, ERL/SEL 89, July 1995.

Smith, Z. K., Dryer, M., and Han, S. M.: Interplanetary shock collisions: Forward with reverse shocks, Astrophys. Space Sci., 119, 337-344, 1986.

Smith, Z., Dryer, M., and Armstrong, M.: Can soft X-rays be used as a proxy for total energy injected by a flare into the interplanetary medium? in: IAU Colloquium 144 on Coronal Structures, (Eds) Rusin, V., Heinsel, P., and Vial, J.-C., Kluwer Acad. Publ., Dordrecht, 267-270, 1993.

Smith, Z., Odstrcil, D., Vandas, M., Fischer, S., Pelant, P., and Dryer, M.: The role of magnetic fields and the heliospheric current sheet in the interplanetary evolution of disturbances caused by solar drivers, in: Solar Drivers of Interplanetary and Terrestrial Disturbances, (Eds) Balasubramanian, K. S., Keil, S. K., and Smart, R. N., Astron. Soc. Pacific Series, 95, 341-349, 1996.

Smith, Z., Dryer, M., Ort, E., and Murtagh, W.: Performance of interplanetary shock prediction models: STOA and ISPM, J. Atm. Solar-Terr. Phys., 62, 1265-1274, 2000.

Stone, E. C., Cohen, C. M. S., Cook, W. R., Cummings, A. C., Gauld, B. W., Kecman, B., Leske, R. A., Mewaldt, R. A., Thayer, M. R., Dougherty, B. I., Grumm, R. L., Milliken, B. D., Radocinski, R. G., Wiedenbeck, M. E., Christian, E. R., Shuman, S., Trexel, H., Von Rosenvinge, T. T., Binns, W. R., Dowkontt, P.,
Epstein, J., Hink, P. L., Klarmann, J., Lijowski, M., and Olevitch, M. A.: The cosmic-ray isotope spectrometer for the Advanced Composition Explorer, Space Sci. Rev., 86, 285-356, 1998.

Svestka, Z.: On 'the solar flare myth' postulated by Gosling, Solar Phys., 160, 53-56, 1995.

Svestka, Z.: Varieties of coronal mass ejections and relation to flares, Space Sci. Rev., 95, 135-146, 2001.

Thompson, B. J., Plunkett, S. P., Gurman, H. B., Newmark, J. S., St. Cyr, O. C., and Michels, D. J.: SOHO/EIT observations of an Earth-directed coronal mass ejection on May 12, 1997, Geophys. Res. Lett., 25 (14), 2465-2468, 1998.

Tylka, A. J., Boberg, P. R., Adams, J. R., Beahm, L. P., Dietrich, W. F., and Kleis, T.: The mean ionic charge state of solar energetic Fe ions above $200 \mathrm{MeV}$ per nucleon, Astrophys. J., 444, L109L113, 1995.

von Rosenvinge, T. T., Barbier, L. M., Karsch, J., Liberman, R., Madden, M. P., Nolan, T., Reames, D. V., Ryan, L., Singh, S., Trexel, H., Winkert, G., Mason, G. M., Hamilton, D. C. and Walpole, P.: The energetic particles: acceleration, composition and transport (EPACT) investigation on the WIND spacecraft, Space Sci. Rev., 71, 155-206, 1995.

Watari, S.-I. and Detman, T. R.: In-situ shock speed and transit shock speed, Ann. Geophysicae, 16, 370-375, 1998.

Webb, D. F., Cheng, C.-C., Dulk, G. A., Edberg, S. J., Martin, S. F., McKenna-Lawlor, S., and McLean, D. J.: Mechanical energy output of the 5 September, 1973 flare, in: Solar Flares, A Monograph from SKYLAB Solar Workshop II (Appendix B), (Ed) Sturrock, P. A., Publ. Colorado Associated University Press, Boulder, Colorado, USA, 1980.

Williams, D. J.: Ion-electron magnetic separation and solid-state detector, NOAA Technical Report, ERL 393-SEL, 40, 1977.

Zwickl, R. D., Doggett, K. A., Sahm, S., Barrett, W. P., Grubb, R. N., Detman, T. R., and Raben, V. J.: The NOAA Real-Time Solar Wind (RTSW) system using ACE data, Space Sci. Rev., 86, 633-648, 1998. 